\title{
HOPF IMAGES AND INNER FAITHFUL REPRESENTATIONS
}

\author{
TEODOR BANICA \\ Département de Mathématiques, Université de Cergy-Pontoise, 2 avenue Chauvin, \\ 95302 Cergy-Pontoise, France \\ e-mail: Teodor.Banica@u-cergy.fr \\ and JULIEN BICHON \\ Laboratoire de Mathématiques, Université Blaise Pascal, Clermont-Ferrand II, \\ Campus des Cézeaux, 63177 Aubière Cedex, France \\ e-mail: Julien.Bichon@math.univ-bpclermont.fr
}

(Received 10 November 2009; revised 2 February 2010; accepted 21 February 2010)

\begin{abstract}
We develop a general theory of Hopf image of a Hopf algebra representation, with the associated concept of inner faithful representation, modelled on the notion of faithful representation of a discrete group. We study several examples, including group algebras, enveloping algebras of Lie algebras, pointed Hopf algebras, function algebras, twistings and cotwistings, and we present a Tannaka duality formulation of the notion of Hopf image.
\end{abstract}

2010 Mathematics Subject Classification. 16W30.

1. Introduction. The aim of this paper is to provide an axiomatization and systematic study of the concept of Hopf image of a Hopf algebra representation, as well as the related concept of inner faithful representation. These notions appeared, under various degrees of generality, in a number of independent investigations: vertex models and related quantum groups $[\mathbf{6}, \mathbf{1 1}, \mathbf{1 2}]$, locally compact quantum groups and their outer actions [20, 27]. These were used extensively in the recent paper [10] in order to study the quantum symmetries of Hadamard matrices and of the corresponding subfactors.

The leading idea is that we want to translate the notion of faithful representation of a discrete group at a Hopf algebra level. Let $\Gamma$ be a group, let $A$ be a $k$-algebra ( $k$ is a field) and consider a representation

$$
\pi: k[\Gamma] \longrightarrow A
$$

There are two possible notions of faithfulness for $\pi$ :

(1) faithfulness of $\pi$ as an algebra map, in which case we simply say that $\pi$ is faithful;

(2) faithfulness of the induced group morphism $\pi_{\mid \Gamma}: \Gamma \longrightarrow A^{\times}$.

It is clear that the first notion is much more restrictive than the second one, and choice of one of these notions as the good one for faithfulness depends on whether one is interested by the algebra $k[\Gamma]$ or rather by the group $\Gamma$ itself.

It is not difficult so see that $\pi_{\mid \Gamma}$ is faithful if and only if $\operatorname{Ker}(\pi) \subset k[\Gamma]$ does not contain any non-zero Hopf ideal. This simple observation leads to a notion of inner faithful representation for arbitrary Hopf algebras: if $H$ a Hopf algebra, we say that a 
representation

$$
\pi: H \longrightarrow A
$$

is inner faithful is $\operatorname{Ker}(\pi)$ does not contain any non-zero Hopf ideal. Of course, $H$ is viewed as the group algebra of a discrete quantum group, and faithfulness refers to this discrete quantum group.

If the representation $\pi$ is not inner faithful, there is, however, a minimal Hopf algebra $H_{\pi}$ that factorizes $\pi$ that we call the Hopf image of $\pi$ (for $H=k[\Gamma]$, we have $\left.k[\Gamma]_{\pi}=k[\pi(\Gamma)]\right)$. The exact universal property of the Hopf image is stated in Section 2. The Hopf image measures how much $\pi$ fails to be faithful in the discrete quantum group sense. In the situation of [10], the Hopf image of the representation of the quantum permutation algebra associated to a complex Hadamard matrix measures the complexity of the quantum invariants of the Hadamard matrix.

A natural concept arising from these considerations is the notion of inner linear Hopf algebra: we say that a Hopf algebra is inner linear if it admits a finitedimensional inner faithful representation. Therefore the problem of inner linearity for Hopf algebras is a generalization of the celebrated linearity problem for discrete groups (see [18, Chapter III, Section 20] for a short overview). More generally, we believe that the study of Hopf images leads to interesting new questions and problems in Hopf algebra theory, as well as in group theory through the study of group duals.

The paper is devoted to a general study of the notion of Hopf image, culminating in a Tannakian formulation, together with the study of several key examples.

The precise contents of the paper are as follows. In Section 2, we give a precise formulation of the concept of Hopf image, we prove the existence of the Hopf image and study some of its basic properties. In Section 3, we get back to the discrete group example discussed in the introduction and also discuss the example of enveloping algebras of Lie algebras. We also introduce the notion of inner linear Hopf algebra. In Section 4, we consider the case of pointed Hopf algebras (thus including quantized enveloping algebras), for which we give a criterion of inner faithfulness for a representation using skew-primitives. As a simple illustration, we examine the vector two-dimensional representation of $U_{q}\left(\mathfrak{s l}_{2}\right)$, which is shown to be inner faithful if and only if $q$ is not a root of unity. Section 5 is devoted to function algebras on algebraic and compact groups. The construction of inner faithful representations is related to the existence of topological generators. These simple examples already show that for co-semi-simple Hopf algebras, inner faithulness is not directly related to injectivity on characters (in contrast with the group algebra situation). In Section 6, we consider the problem of constructing inner faithful representations for Hopf algebras with twisted coproduct or twisted product. We show that the cotwist (2-cocycle deformation) of an inner linear Hopf algebra for which $S^{2}$ is an inner automorphism is still inner linear if the 2-cocycle is induced by a finite-dimensional quotient. In particular, the multiparametric 2-cocycle deformations (cotwists) of $\mathcal{O}\left(\mathrm{GL}_{n}(\mathbb{C})\right)$ at roots of unity are inner linear. Section 7 is devoted to the study of tensor and free products of Hopf images. The question of knowing whether a tensor product of inner faithful representations is still inner faithful leads to the notion of projectively inner faithful representation. Section 8 is devoted to Tannaka duality type results, which provide so far the best inner faithfulness criteria for a representation of a compact Hopf algebra (i.e. a Hopf algebra associated to a compact quantum group). These are used in Section 9 to 
describe examples of inner faithful representations of compact Hopf algebras having all their simple comodules of dimension smaller than 2 .

Notations and conventions. We work in general over a fixed field $k$. We assume that the reader has some familiarity with Hopf algebras, for which the textbooks [21] or [26] are convenient. Our terminology and notation are the standard ones: in particular, for a Hopf algebra, $\Delta, \varepsilon$ and $S$ denote the comultiplication, counit and antipode, respectively.

2. Construction of the Hopf image and basic properties. In this section, we give the precise formulation of the concept of Hopf image, prove its existence and study of some of its basic properties. The case of Hopf $*$-algebras is also considered at the end of the section.

2.1. Construction of the Hopf image. First, let us give a precise formulation of the notion of Hopf image of a representation. Let $H$ be a Hopf algebra over a field $k$. As usual, a representation of $H$ on an algebra $A$ is an algebra morphism $\pi: H \longrightarrow A$.

Let us say that a factorization of $\pi$ is a triple $(L, q, \varphi)$, where $L$ is a Hopf algebra, $q: H \longrightarrow L$ is a surjective Hopf algebra map and $\varphi: L \longrightarrow A$ is a representation, with the decomposition $\pi=\varphi \circ q$. We define in a straightforward manner the category of factorizations of $\pi$, and the Hopf image of $\pi$ is defined to be the final object in this category (hence we can also say that this is a minimal factorization).

THEOREM 2.1. Let $\pi: H \longrightarrow A$ be a representation of a Hopf algebra $H$ on an algebra A. Then $\pi$ has a Hopf image: there exists a Hopf algebra $H_{\pi}$ together with a surjective Hopf algebra map $p: H \longrightarrow H_{\pi}$ and a representation $\tilde{\pi}: H_{\pi} \longrightarrow A$, such that $\pi=\tilde{\pi} \circ p$, and such that if $(L, q, \varphi)$ is another factorization of $\pi$, there exists a unique Hopf algebra map $f: L \longrightarrow H_{\pi}$ such that $f \circ q=p$ and $\tilde{\pi} \circ f=\varphi$.

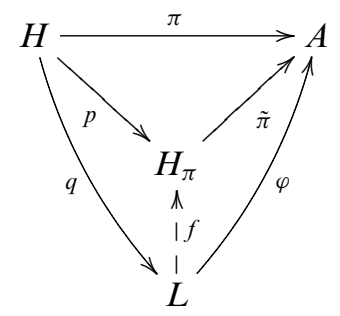

Proof. Let $I_{\pi}$ be sum of all the Hopf ideals contained in $\operatorname{Ker}(\pi)$. It is clear that $I_{\pi}$ is a Hopf ideal and is the largest Hopf ideal contained in $\operatorname{Ker}(\pi)$. Let $H_{\pi}=H / I_{\pi}$ and let $p: H \longrightarrow H_{\pi}$ be the canonical surjection. Since $I_{\pi} \subset \operatorname{Ker}(\pi)$, there exists a unique algebra map $\tilde{\pi}: H_{\pi} \longrightarrow A$ such that $\tilde{\pi} \circ p=\pi$.

Let $(L, q, \varphi)$ be a factorization of $\pi$. Then $\operatorname{Ker}(q)$ is a Hopf ideal contained in $\operatorname{Ker}(\pi)$ and hence $\operatorname{Ker}(q) \subset I_{\pi}$. Hence there exists a unique Hopf algebra map $f$ : $L \longrightarrow H_{\pi}, q(x) \longmapsto p(x)$, satisfying $f \circ q=p$ and $\varphi \circ f=\tilde{\pi}$, and thus $H_{\pi}$ has the required universal property.

As noticed by the referee, the notion of Hopf image is formally dual to the notion of Hopf sub-algebra generated by the image of a coalgebra morphism. 
The above proof uses the Hopf ideal $I_{\pi}$, the largest Hopf ideal contained in $\operatorname{Ker}(\pi)$. This Hopf ideal is constructed in a very abstract manner, and it is useful for several purposes to have a more concrete description of $I_{\pi}$, that we give now.

Let $F$ be the free monoid generated by the set $\mathbb{N}$, with its generators denoted $\alpha_{0}, \alpha_{1}, \ldots$ and its unit element denoted 1 . We denote by $\tau: F \longrightarrow F$ the unique monoid anti-morphism such that $\tau\left(\alpha_{i}\right)=\alpha_{i+1}$ for all $i$.

To any element $g \in F$, we associate an algebra $A^{g}$, defined inductively on the length of $g$ as follows. We put $A^{1}=k, A^{\alpha_{k}}=A$ if $k$ is even and $A^{\alpha_{k}}=A^{\text {op }}$ if $k$ is odd. Now for $g, h \in F$ with $l(g) \geq 1$ and $l(h) \geq 1$, we put $A^{g h}=A^{g} \otimes A^{h}$.

Now we associate an algebra morphism $\pi^{g}: H \longrightarrow A^{g}$ to any $g \in F$, again by induction on the length of $g$. We put $\pi^{1}=\varepsilon, \pi^{\alpha_{k}}=\pi \circ S^{k}$, and for $g, h \in F$ with $l(g) \geq 1$ and $l(h) \geq 1$, we put $\pi^{g h}=\left(\pi^{g} \otimes \pi^{h}\right) \circ \Delta$.

Proposition 2.2. Let $\pi: H \longrightarrow$ A be a representation and let $I_{\pi}$ be the largest Hopf ideal contained in $\operatorname{Ker}(\pi)$. We have

$$
I_{\pi}=\bigcap_{g \in F} \operatorname{Ker}\left(\pi^{g}\right) \subset H .
$$

The proof of Proposition 2.2 uses several lemmas. We put $J_{\pi}=\bigcap_{g \in F} \operatorname{Ker}\left(\pi^{g}\right) \subset H$. By construction $J_{\pi}$ is an ideal in $H$, and we wish to prove now that $J_{\pi}$ is a Hopf ideal. The following result ensures that it is a co-ideal.

LEMMA 2.3. Let $C_{\pi}$ be the linear subspace in $H^{*}$ generated by the elements

$$
\left\{\psi \circ \pi^{g}, g \in F, \psi \in A^{g^{*}}\right\} \subset H^{*} .
$$

Then $C_{\pi}$ is a sub-algebra of $H^{*}$, and $J_{\pi}=C_{\pi}^{\perp}$. In particular, $J_{\pi}$ is a co-ideal in $H$.

Proof. For $\psi, \phi \in H^{*}$ and $g, h \in F$, we have

$$
\left(\psi \circ \pi^{g}\right) \cdot\left(\phi \circ \pi^{h}\right)=\left(\left(\psi \circ \pi^{g}\right) \otimes\left(\phi \circ \pi^{h}\right)\right) \circ \Delta=(\psi \otimes \phi) \circ \pi^{g h}
$$

and since $\varepsilon \in C_{\pi}$, we conclude that $C_{\pi}$ is a sub-algebra of $H^{*}$. It is clear that $C_{\pi}^{\perp}=J_{\pi}$, and we conclude that $J_{\pi}$ is a co-ideal by [26, Proposition 1.4.6].

Lemma 2.4. For all $g \in F$, there exists a linear isomorphism $R_{g}: A^{\tau(g)} \longrightarrow A^{g}$ such that $\pi^{g} \circ S=R_{g} \circ \pi^{\tau(g)}$. In particular, $S\left(J_{\pi}\right) \subset J_{\pi}$, and $J_{\pi}$ is a Hopf ideal in $H$.

Proof. We prove the result by induction on $l(g)$. For $l(g)=0$, this is clear and for $l(g)=1$, we can take $R_{g}=\mathrm{id}_{A}$ since $\pi^{\tau\left(\alpha_{k}\right)}=\pi^{\alpha_{k+1}}=\pi^{\alpha_{k}} \circ S$. So assume that $l(g) \geq 1$, so that $g=h h^{\prime}$, with $l(h)=l(g)-1$ and $l\left(h^{\prime}\right)=1$ and that the result is proved for elements of length $<l(g)$. We put

$$
R_{g}=R_{h h^{\prime}}=c_{h^{\prime}, h} \circ\left(R_{h^{\prime}} \otimes R_{h}\right): A^{\tau(g)}=A^{\tau\left(h^{\prime}\right) \tau(h)}=A^{\tau\left(h^{\prime}\right)} \otimes A^{\tau(h)} \longrightarrow A^{h} \otimes A^{h^{\prime}}=A^{g},
$$

where $c_{h^{\prime}, h}: A^{h^{\prime}} \otimes A^{h} \longrightarrow A^{h} \otimes A^{h^{\prime}}$ is the usual symmetry (flip) map. For $x$ in $H$, we have

$$
\begin{aligned}
\pi^{g} \circ S(x) & =\pi^{h h^{\prime}} \circ S(x)=\pi^{h}\left(S\left(x_{2}\right)\right) \otimes \pi^{h^{\prime}}\left(S\left(x_{1}\right)\right)=\left(\left(R_{h} \circ \pi^{\tau(h)}\right)\left(x_{2}\right)\right) \otimes\left(\left(R_{h^{\prime}} \circ \pi^{\tau\left(h^{\prime}\right)}\right)\left(x_{1}\right)\right) \\
& =R_{h h^{\prime}} \circ\left(\pi^{\tau\left(h^{\prime}\right)} \otimes \pi^{\tau(h)}\right) \circ \Delta(x)=R_{g} \circ \pi^{\tau(g)}(x),
\end{aligned}
$$

which proves the result. The last assertion is then clear. 
The following lemma finishes the proof of Proposition 2.2.

Lemma 2.5. Let $\pi: H \longrightarrow$ A be an algebra map, let $q: H \longrightarrow$ L be a Hopf algebra map, and let $\varphi: L \longrightarrow A$ be an algebra map with $\pi=\varphi \circ q$. Then $\operatorname{Ker}(q) \subset J_{\pi}$, and hence any Hopf ideal contained in $\operatorname{Ker}(\pi)$ is contained in $J_{\pi}$.

Proof. Let us show that

$$
\pi^{g}=\varphi^{g} \circ q, \forall g \in F
$$

with the same notation for $\varphi$ as the one for $\pi$. We show this by induction on $l(g)$. This is true if $l(g)=0$ since $q$ is a coalgebra map and if $l(g)=1$, we have $\pi^{\alpha_{k}}=$ $\pi \circ S^{k}=\varphi \circ q \circ S^{k}=\varphi \circ S^{k} \circ q=\varphi^{\alpha_{k}} \circ q$. Assume now that $l(g)>1$, so that $g=h h^{\prime}$ with $l(g)>l(h) \geq 1$ and $l(g)>l\left(h^{\prime}\right) \geq 1$, and that the result is proved for elements of length $<l(g)$. Then

$$
\pi^{h h^{\prime}}=\left(\pi^{h} \otimes \pi^{h^{\prime}}\right) \circ \Delta=\left(\varphi^{h} \otimes \varphi^{h^{\prime}}\right) \circ(q \otimes q) \circ \Delta=\left(\varphi^{h} \otimes \varphi^{h^{\prime}}\right) \circ \Delta \circ q=\varphi^{h h^{\prime}} \circ q,
$$

and this proves our assertion by induction. Hence we have $\operatorname{Ker}(q) \subset J_{\pi}$. Any Hopf ideal is the kernel of a Hopf algebra map, and hence the last assertion follows.

REMARK 2.6. The notion of Hopf image considered here is in general different from the one given in [2], Definition 1.2.0, which refers to the category of Hopf algebras. Our definition of Hopf image uses the larger category of algebras.

2.2. Inner faithful representations. The following definition was already given in the introduction.

Definition 2.7. Let $\pi: H \longrightarrow A$ be a representation of a Hopf algebra $H$ on an algebra $A$. We say that $\pi$ is inner faithful if $\operatorname{Ker}(\pi)$ does not contain any non-zero Hopf ideal.

We have several equivalent formulations for inner faithfulness.

Proposition 2.8. Let $\pi: H \longrightarrow A$ be a representation of a Hopf algebra $H$ on an algebra $A$. The following assertions are equivalent.

(1) $\pi$ is inner faithful.

(2) $\bigcap_{g \in F} \operatorname{Ker}\left(\pi^{g}\right)=(0)$.

(3) The Hopf algebra morphism $p: H \longrightarrow H_{\pi}$ in Theorem 2.1 is an isomorphism.

(4) If $(L, q, \varphi)$ is any factorization of $\pi$, then $q$ is an isomorphism.

The equivalence between these assertions follows from the previous considerations and Proposition 2.2. Under a special assumption, we also have another equivalent criterion for inner faithfulness.

Proposition 2.9. Let $\pi: H \longrightarrow A$ be a representation of a Hopf algebra $H$ on an algebra A such that $\operatorname{Ker}(\pi)$ is $S$-stable: $S(\operatorname{Ker}(\pi)) \subset \operatorname{Ker}(\pi)$. Then $\pi$ is inner faithful if and only if $(0)$ is the only bi-ideal contained in $\operatorname{Ker}(\pi)$.

Proof. Assume that $\pi$ is inner faithful. Let $I \subset \operatorname{Ker}(\pi)$ be a bi-ideal. Then $J=$ $\sum_{k \in \mathbb{N}} S^{k}(I)$ is a co-ideal stable under the antipode with $J \subset \operatorname{Ker}(\pi)$. Let $J^{\prime}$ be the ideal generated by $J$ : this is a bi-ideal with $S\left(J^{\prime}\right) \subset J^{\prime}$, and hence $J^{\prime}$ is a Hopf ideal contained in $\operatorname{Ker}(\pi)$. Hence, by the previous proposition, we have $J=J^{\prime}=(0)$. The converse assertion is immediate. 
We also have a characterization of the Hopf image using inner faithfulness, which can be useful in some contexts (e.g. see the proof of Proposition 3.3).

Proposition 2.10. Let $\pi: H \longrightarrow$ A be a representation of a Hopf algebra $H$ on an algebra $A$, and let $\left(H_{\pi}, p, \tilde{\pi}\right)$ be the Hopf image of $\pi$. Then $\tilde{\pi}$ is inner faithful. Conversely if $(L, q, \varphi)$ is a factorization of $\pi$ such that $\varphi$ is inner faithful, then $L \simeq H_{\pi}$.

Proof. Let $(L, q, \varphi)$ be a factorization of $\tilde{\pi}: H_{\pi} \longrightarrow A$ : we have $\varphi \circ q=\tilde{\pi}$, and hence $\varphi \circ(q \circ p)=\pi$. Thus there exists a Hopf algebra map $f: L \longrightarrow H_{\pi}$ such that $f \circ q \circ p=p$. Hence $f \circ q=\mathrm{id}_{H_{\pi}}, q$ is injective and is an isomorphism. By Proposition 2.8 we conclude that $\tilde{\pi}$ is inner faithful.

Now let $(L, q, \varphi)$ be a factorization of $\pi$ with $\varphi$ inner faithful. By the universal property of the Hopf image, there exists a surjective Hopf algebra map $f: L \longrightarrow H_{\pi}$ such that $\tilde{\pi} \circ f=\varphi$. But then $\left(H_{\pi}, f, \tilde{\pi}\right)$ is a factorization of $\varphi$, and Proposition 2.8 ensures that $f$ is an isomorphism.

REMARK 2.11. Let $\pi: H \longrightarrow A$ be a representation of a Hopf algebra $H$ on an algebra $A$ and let $\theta: A \longrightarrow B$ be an algebra isomorphism. The universal property of the Hopf image ensures that we have a Hopf algebra isomorphism $H_{\theta \circ \pi} \simeq H_{\pi}$, and, in particular, $\theta \circ \pi$ is inner faithful if and only if $\pi$ is inner faithful.

When considering Hopf algebra maps, Hopf images are usual images and inner faithfulness is equivalent to faithfulness.

Proposition 2.12. Let $H$ and $A$ be Hopf algebras and $\pi: H \longrightarrow A$ be a Hopf algebra map. Then the Hopf image of $\pi$ is $\pi(H)$, and $\pi$ is inner faithful if and only if it is faithful.

Both assertions are immediate, since $\operatorname{Ker}(\pi)$ is a Hopf ideal.

Of course, one cannot expect that an inner faithful representation $H \longrightarrow A$ will transmit all the algebra properties of the algebra $A$ to the algebra $H$. This is true, however, for commutativity.

Proposition 2.13. Let $\pi: H \longrightarrow A$ be a representation of a Hopf algebra $H$ on a commutative algebra A. Then the Hopf image $H_{\pi}$ is a commutative algebra, and hence if $\pi$ is inner faithful, then $H$ is also commutative.

Proof. Since $A$ is commutative, the ideal of $H$ generated by the commutators of elements of $H$ is contained in $\operatorname{Ker}(\pi)$. But the commutator ideal is also a Hopf ideal, so is contained in $I_{\pi}$, and hence $H_{\pi}$ is commutative.

2.3. Hopf $*$-algebras. In this subsection, we assume that $k=\mathbb{C}$, and we consider Hopf $*$-algebras. In this case the construction of the previous subsection has to be slightly modified.

Let us begin by recalling the appropriate language. First, a Hopf $*$-algebra is a Hopf algebra $H$ which is a $*$-algebra and such that the comultiplication $\Delta: H \longrightarrow H \otimes H$ is a $*$-algebra map. It follows that the counit is a $*$-morphism and that the antipode is bijective, and its inverse satisfies $S^{-1}(x)=S\left(x^{*}\right)^{*}$. A $*$-representation of $H$ on a $*$-algebra is a $*$-algebra map $H \longrightarrow A$.

The formulation of the problem of the existence of a Hopf $*$-image is the same as in the introduction, adding ' $*$ ' where needed. In this framework, Theorem 2.1 has the following form. 
THEOREM 2.14. Let $\pi: H \longrightarrow$ A be a $*$-representation of a Hopf $*$-algebra $H$ on an *-algebra A. Then $\pi$ has a Hopf $*$-image: there exists a Hopf $*$-algebra $H_{\pi}$ together with a surjective Hopf $*$-algebra map $p: H \longrightarrow H_{\pi}$ and a $*$-representation $\tilde{\pi}: H_{\pi} \longrightarrow A$, such that $\pi=\tilde{\pi} \circ p$, and such that if $(L, q, \varphi)$ is another $*$-factorization of $\pi$, there exists a unique Hopf $*$-algebra map $f: L \longrightarrow H_{\pi}$ such that $f \circ q=p$ and $\tilde{\pi} \circ f=\varphi$.

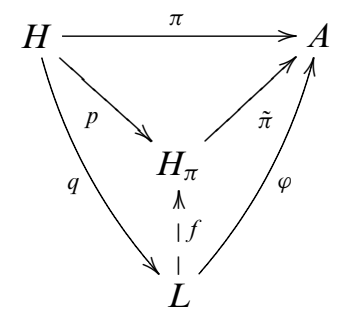

Proof. Similarly to the proof of Theorem 2.1, one constructs $I_{\pi}^{+}$, the largest Hopf *-ideal contained in $\operatorname{Ker}(\pi)$. The explicit construction of $I_{\pi}^{+}$is as follows. Let $F^{+}$be the free monoid generated by the set $\mathbb{Z}$. Similarly to the construction in the previous subsection, to any $g \in F^{+}$, we associate an algebra $A^{g}$ and a representation $\pi^{g}: H \longrightarrow$ $A^{g}$, using the inverse of the antipode when needed. Then, similarly to the proof of Proposition 2.2, we show that

$$
I_{\pi}^{+}=\bigcap_{g \in F^{+}} \operatorname{Ker}\left(\pi^{g}\right) \subset H .
$$

The details are left to the reader (to show that $\bigcap_{g \in F^{+}} \operatorname{Ker}\left(\pi^{g}\right)$ is $*$-stable, one uses the formula $S \circ *=* \circ S^{-1}$ ).

REMARK 2.15. We have used the same notation $H_{\pi}$ for the Hopf $*$-image of a *-representation $\pi: H \longrightarrow A$ and its Hopf image. In general, it seems possible that two notions might not coincide, although we do not have an explicit example. This should cause no confusion: unless specifically notified, when we have such a $*$-representation, the notation $H_{\pi}$ will always denote the Hopf $*$-image.

We will say that a $*$-representation $\pi: H \longrightarrow A$ of a Hopf $*$-algebra $H$ on an *-algebra $A$ is inner faithful if $\operatorname{Ker}(\pi)$ does not contain any non-zero Hopf $*$-ideal (similarly to Proposition 2.8 there are several equivalent characterizations). Again it seems to be possible that such a $*$-representation be inner faithful as a $*$-representation, but not as a representation. However, the two notions coincide when $S^{2}=\mathrm{id}_{H}$ (or more generally if some power of $S^{2}$ is an inner automorphism).

REMARK 2.16. The proof of Theorem 2.14 also shows that Hopf images exist in the category of Hopf algebras having a bijective antipode (more precisely, the Hopf ideal $I_{\pi}^{+}$is the largest Hopf ideal with $S\left(I_{\pi}^{+}\right)=I_{\pi}^{+}$contained in $\operatorname{Ker}(\pi)$ ), and the Hopf image in this category coincides with the Hopf $*$-image. Note that the construction of Theorem 2.1 does not give the Hopf image in the category of Hopf algebras with bijective antipode, since there exist quotients of Hopf algebras with bijective antipode that do not have a bijective antipode (see e.g. [25]).

We now turn to compact Hopf algebras: these are the Hopf $*$-algebras having all their finite-dimensional comodules equivalent to unitary ones (see [19], these are called 
CQG algebras there), and are the algebras of representative functions on compact quantum groups.

THEOREM 2.17. Let $\pi: H \longrightarrow$ A be a $*$-representation of a compact Hopf algebra $H$ on $a *$-algebra $A$. Then the Hopf $*$-image $H_{\pi}$ is a compact Hopf algebra, and hence Hopf $*$-images exist in the category of compact Hopf algebras.

Proof. It is clear from Theorem 27, Section 11.3 in [19], that a quotient of a compact Hopf algebra is again a compact Hopf algebra. Thus since we have a surjective Hopf *-algebra map $H \longrightarrow H_{\pi}$, we conclude that $H_{\pi}$ is a compact Hopf algebra.

3. Classical examples: group algebras and Lie algebras. In this section, we get back to the motivating examples for the notion of inner faithfulness: group algebras and universal enveloping algebras of Lie algebras.

3.1. Group algebras. The following result is announced in the introduction. Its origin goes back to Proposition 2.2 in [6].

Proposition 3.1. Let $\Gamma$ be a group, let $A$ be an algebra and let $\pi: k[\Gamma] \longrightarrow A$ be an algebra map. Then we have

$$
k[\Gamma]_{\pi} \simeq k[\pi(\Gamma)] \simeq k\left[\Gamma / \operatorname{Ker}\left(\pi_{\mid \Gamma}\right)\right] .
$$

In particular, $\pi$ is inner faithful if and only if the group morphism $\pi_{\mid \Gamma}: \Gamma \longrightarrow A^{\times}$is injective (faithful).

Proof. Let $(L, q, \varphi)$ be a factorization of $\pi$. Then $L$ is the group algebra $k[q(\Gamma)]$, and we have a group factorization

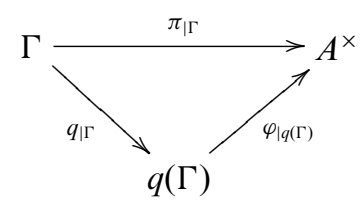

Hence, we have $q(\Gamma) \subset \pi(\Gamma)$, and this induces the appropriate Hopf algebra map $k[q(\Gamma)]=L \longrightarrow k[\pi(\Gamma)]$.

The above result motivates the following definition.

Definition 3.2. A Hopf algebra $H$ is said to be inner linear if there exists an inner faithful representation $\pi: H \longrightarrow A$ into a finite-dimensional algebra $A$.

Indeed the group algebra $k[\Gamma]$ is inner linear if and only the group $\Gamma$ is linear (over $k$ ). It is clear that a Hopf algebra is inner linear if and only if it contains an ideal of finite codimension that does not contain non-zero Hopf ideals.

3.2. Lie algebras. Let $\mathfrak{g}$ be a Lie algebra, and let $U(\mathfrak{g})$ be its universal enveloping algebra. Algebra maps $U(\mathfrak{g}) \longrightarrow A$ correspond exactly to Lie algebra maps $\mathfrak{g} \longrightarrow$ $\operatorname{gl}(A)$, where $\mathfrak{g l}(A)$ is the Lie algebra associated to $A$ : as a vector space $\mathfrak{g l}(A)=A$ and the Lie bracket is defined by $[a, b]=a b-b a, \forall a, b \in A$. The Hopf image is described as follows in characteristic zero. 
Proposition 3.3. Let $\mathfrak{g}$ be a Lie algebra over a characteristic zero field, let $A$ be an algebra and let $\pi: U(\mathfrak{g}) \longrightarrow A$ be an algebra map. Then we have

$$
U(\mathfrak{g})_{\pi} \simeq U(\pi(\mathfrak{g})) \simeq U\left(\mathfrak{g} / \operatorname{Ker}\left(\pi_{\mid \mathfrak{g}}\right)\right)
$$

In particular, $\pi$ is inner faithful if and only if the Lie algebra map $\pi_{\mid \mathfrak{g}}: \mathfrak{g} \longrightarrow \mathfrak{g l}(A)$ is injective (faithful). Thus the Hopf algebra $U(\mathfrak{g})$ is inner linear if and only if $\mathfrak{g}$ is finite-dimensional.

Proof. Let us first show if $\pi_{\mid \mathfrak{g}}: \mathfrak{g} \longrightarrow \mathfrak{g l}(A)$ is injective, then $\pi: U(\mathfrak{g}) \longrightarrow A$ is inner faithful. The space of primitive elements $\mathcal{P}(U(\mathfrak{g}))$ equals $\mathfrak{g}$ by the characteristic zero assumption and 1 is the only group-like in $U(\mathfrak{g})$ (see e.g. [21, Proposition 5.5.3]). Thus the canonical map $p: U(\mathfrak{g}) \longrightarrow U(\mathfrak{g})_{\pi}$ is injective on primitive elements, and since the Hopf algebra $U(\mathfrak{g})$ is pointed, we use Corollary 5.4.7 in [21] to conclude that $p$ is injective, and hence $\pi$ is inner faithful.

In general, we have a Hopf algebra factorization

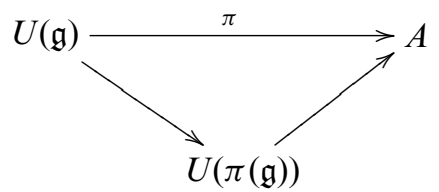

The algebra map on the right is inner faithful by the previous discussion, and hence by Proposition 2.10 we have the announced isomorphism $U(\mathfrak{g})_{\pi} \simeq U(\pi(\mathfrak{g})) \simeq$ $U\left(\mathfrak{g} / \operatorname{Ker}\left(\pi_{\mid \mathfrak{g}}\right)\right)$. Finally, if $\pi$ is inner faithful, it induces an isomorphism $U(\mathfrak{g}) \simeq$ $U(\pi(\mathfrak{g}))$, which induces an isomorphism between the Lie algebras of primitive elements, and hence between $\mathfrak{g}$ and $\pi(\mathfrak{g}): \pi_{\mid \mathfrak{g}}$ is injective.

If $U(\mathfrak{g})$ is inner linear, then $\mathfrak{g}$ is finite-dimensional by the previous discussion. The converse follows from Ado's Theorem: a finite-dimensional Lie algebra $\mathfrak{g}$ has a faithful finite-dimensional representation $\mathfrak{g} \longrightarrow \mathfrak{g l}_{n}(k)$ for some $n$.

In fact, the main argument we have used for the proof of this proposition, Corollary 5.4.7 in [21], a result due independently to Takeuchi and Radford, is valid for arbitrary pointed Hopf algebras. We use it in a similar manner in the next section to get an inner faithfulness criterion for representations of arbitrary pointed Hopf algebras.

4. Pointed Hopf algebras. After the motivating examples of group algebras and enveloping algebras of Lie algebras, the next natural step is the study of pointed Hopf algebras. These have been much studied in recent years (see e.g. [19] for quantized enveloping algebras of Lie algebras and [3] for the finite-dimensional case). In this section, we study the inner faithfulness of a representation of a pointed Hopf algebra. The criterion that we give unifies those given in the previous section.

Recall that a Hopf algebra $H$ is said to be pointed if all its simple comodules are one-dimensional, hence corresponding to group-like elements. The group of group-like elements of $H$ is denoted by $\mathrm{G}(H)$. An element $x \in H$ is said to be $(g, h)$-primitive for $g, h \in \mathrm{G}(H)$ if

$$
\Delta(x)=g \otimes x+x \otimes h .
$$

The space of $(g, h)$-primitive elements is denoted $\mathcal{P}_{g, h}(H)$. 
TheOREM 4.1. Let $\pi: H \longrightarrow$ A be a representation of a pointed Hopf algebra $H$ on an algebra $A$. The following assertions are equivalent.

(1) $\pi$ is inner faithful.

(2) $\forall g \in \mathrm{G}(H)$, the restriction $\pi_{\mid \mathcal{P}_{g, 1}(H)}: \mathcal{P}_{g, 1}(H) \longrightarrow A$ is injective.

(3) $\forall g \in \mathrm{G}(H)$, the restriction $\pi_{\mid \mathcal{P}_{1, g}(H)}: \mathcal{P}_{1, g}(H) \longrightarrow A$ is injective.

Proof. We begin by showing that $\pi$ is inner faithful if and only if for all $g, h \in \mathrm{G}(H)$, the restriction $\pi_{\mid \mathcal{P}_{g, h}(H)}: \mathcal{P}_{g, h}(H) \longrightarrow A$ is injective. Assume that $\pi$ is inner faithful. Let $x \in \mathcal{P}_{g, h}(H)$ be such that $\pi(x)=0$ and let $I$ be the ideal of $H$ generated by $x$. It is clear from the identities

$$
\Delta(x)=g \otimes x+x \otimes h \quad \text { and } \quad S(x)=-g^{-1} x h^{-1}
$$

that $I$ is a Hopf ideal, and since $I$ is contained in $\operatorname{Ker}(\pi)$, we get $I=0$ and $x=0$.

Conversely, assume that $\pi$ is injective on each space of primitives. Then so is $p$ : $H \longrightarrow H_{\pi}$, and hence by Corollary 5.4.7 in [21], we conclude that $p$ is an isomorphism and that $\pi$ is inner faithful.

For $x \in \mathcal{P}_{g, h}(H)$, we have $g^{-1} x \in \mathcal{P}_{1, g^{-1} h}(H)$ and $h^{-1} x \in \mathcal{P}_{h^{-1} g, 1}(H)$. Hence if (2) or (3) holds, then $\pi$ is injective when restricted to each space of primitives, and the previous discussion shows that $\pi$ is inner faithful.

The above criterion applies, in particular, to the quantized universal enveloping algebra $U_{q}(\mathfrak{g})$ of a semi-simple Lie algebra $\mathfrak{g}$, for which descriptions of skew-primitives are available $[\mathbf{1 4}, \mathbf{2 2}]$. As a simple illustration, let us have a look at the vector representation of $U_{q}\left(\mathfrak{s l}_{2}(\mathbb{C})\right)$.

THEOREM 4.2. Let $\pi: U_{q}\left(\mathfrak{s l}_{2}(\mathbb{C})\right) \longrightarrow M_{2}(\mathbb{C})$ be the two-dimensional vector representation of $U_{q}\left(\mathfrak{s l}_{2}(\mathbb{C})\right)$. Then $\pi$ is inner faithful if and only if $q$ is not a root of unity, and hence $U_{q}\left(\mathfrak{s l}_{2}(\mathbb{C})\right)$ is inner linear if $q$ is not a root of unity.

Proof. Recall first that for $q \in \mathbb{C}, q^{2} \neq 1$ and $q \neq 0$, the algebra $U_{q}=U_{q}\left(\mathfrak{s l}_{2}(\mathbb{C})\right)$ is presented by the generators $E, F, K, K^{-1}$, submitted to the relations

$$
K K^{-1}=1=K^{-1} K, K E=q^{2} E K, K F=q^{-2} F K,[E, F]=\frac{K-K^{-1}}{q-q^{-1}}
$$

and its comultiplication is defined by

$$
\Delta(E)=1 \otimes E+E \otimes K, \quad \Delta(F)=K^{-1} \otimes F+F \otimes 1, \quad \Delta(K)=K \otimes K .
$$

The vector representation $\pi: U_{q} \longrightarrow M_{2}(\mathbb{C})$ is defined by

$$
\pi(K)=\left(\begin{array}{cc}
q & 0 \\
0 & q^{-1}
\end{array}\right), \pi(E)=\left(\begin{array}{ll}
0 & 1 \\
0 & 0
\end{array}\right), \pi(K)=\left(\begin{array}{ll}
0 & 0 \\
1 & 0
\end{array}\right) .
$$

We have $\mathrm{G}\left(U_{q}\right)=\left\{K^{n}, n \in \mathbb{Z}\right\} \simeq \mathbb{Z}$. If $q$ is not a root of unity we have

$$
\mathcal{P}_{1, K}\left(U_{q}\right)=\mathbb{C}(1-K) \oplus \mathbb{C} E \oplus \mathbb{C} F K \text {, and for } m \neq 1, \mathcal{P}_{1, K^{m}}\left(U_{q}\right)=\mathbb{C}\left(1-K^{m}\right) .
$$

It is a direct computation to check that the conditions of Theorem 4.1 are fulfilled, and hence $\pi$ is inner faithful. If $q$ is a root of unity, then $\pi$ is not injective on the group-like elements and hence is not inner faithful. 
REMARK 4.3. If $q$ is a root of unity, one can show that the Hopf image of the above representation $\pi$ is the (finite-dimensional) Hopf algebra $u_{q}\left(\mathfrak{s} l_{2}(\mathbb{C})\right.$ ).

5. Function algebras. In this section, we study Hopf images for various function algebras: polynomial functions on algebraic groups and representative functions on compact groups. The idea for the computation of the Hopf image goes back to [6], but we are a little bit more general here. These simple examples are already interesting for testing the possibility of generalizing representation theoretic properties of discrete group algebras to arbitrary Hopf algebras.

Proposition 5.1. Let $G$ be a linear algebraic group over an algebraically closed field of characteristic zero and let $\pi: \mathcal{O}(G) \longrightarrow A$ be a representation on an algebra $A$. Assume that the algebra $\pi(A)$ is reduced, so that $\pi(A) \simeq \mathcal{O}(X)$ for an affine algebraic set $X$ and that the algebra map $\mathcal{O}(G) \longrightarrow \pi(A) \simeq \mathcal{O}(X)$ is induced by a polynomial map $v: X \longrightarrow G$. Then $\mathcal{O}(G)_{\pi} \simeq \mathcal{O}(\overline{\langle v(X)\rangle})$, where $\overline{\langle v(X)\rangle}$ is the Zariski closure in $G$ of the subgroup generated by $v(X)$.

Proof. By the assumption and Remark 2.11 we may assume that $\pi: \mathcal{O}(G) \longrightarrow$ $\mathcal{O}(X)$ is induced by an injective polynomial map $v: X \longrightarrow G$. The injections $X \rightarrow$ $\overline{\langle v(X)\rangle} \subset G$ yield a factorization of $\pi$. Now let $(L, q, \varphi)$ be a factorization of $\pi$. Then $L$ is finitely generated and is reduced by Cartier's theorem ( $k$ has characteristic zero), hence we can assume that $L=\mathcal{O}(H)$ for a linear algebraic group $H$, and that $q$ and $\rho$ are induced by polynomial maps $X \rightarrow H \subset G$ whose composition is $v$. Hence $\overline{\langle v(X)\rangle} \subset H$, and this gives the required Hopf algebra map $\mathcal{O}(H) \longrightarrow \mathcal{O}(\overline{\langle v(X)\rangle})$.

EXAMPLE 5.2. Let $G$ be a linear algebraic group over an algebraically closed field of characteristic zero and let $g_{1}, \ldots, g_{n} \in G$. The algebra map

$$
\begin{aligned}
\mathcal{O}(G) & \longrightarrow k^{n} \\
f & \longmapsto\left(f\left(g_{1}\right), \ldots, f\left(g_{n}\right)\right)
\end{aligned}
$$

has $\mathcal{O}\left(\overline{\left\langle g_{1}, \ldots, g_{n}\right\rangle}\right)$ as Hopf image and is inner faithful if and only if $G=\overline{\left\langle g_{1}, \ldots, g_{n}\right\rangle}$.

Proposition 5.3. If $G$ is a linear algebraic group over an algebraically closed field of characteristic zero, there exists an inner faithful representation $\pi: \mathcal{O}(G) \longrightarrow k^{n}$ for some $n \in \mathbb{N}^{*}$, and hence the Hopf algebra $\mathcal{O}(G)$ is inner linear.

Proof. By the previous example it is enough to show that there exists $g_{1}, \ldots, g_{n} \in G$ that generate $G$ as an algebraic group: $G=\overline{\left\langle g_{1}, \ldots, g_{n}\right\rangle}$. We can assume that $G$ is connected since if $G_{0}$ is the neutral component of $G$, the group $G / G_{0}$ is finite. The Levi decomposition of $G$ as a semi-direct product of a reductive group and a unipotent one reduces the problem to the case when $G$ is unipotent or $G$ is reductive. If $G$ is unipotent, we can use an induction $\operatorname{argument}$ on $\operatorname{dim}(G)$ to prove the result, since all the subgroups of $G$ are unipotent (and hence connected by the characteristic zero assumption). If $G$ is reductive, we have $G=\overline{B B^{\prime}}$ where $B, B^{\prime}$ are Borel subgroups containing a maximal torus $T$. We have $B=T U$ and $B=T U^{\prime}$ with $U, U^{\prime}$ unipotent. Therefore it just remains to treat the torus case, for which the result is clear.

The compact group case is worked out similarly. Let $G$ be a compact group. The Hopf $*$-algebra of representative functions on $G$ is denoted by $\mathcal{R}(G)$ : this a 
dense $*$-sub-algebra of $\mathrm{C}(G)$ by the Peter-Weyl theorem, and moreover $\mathrm{C}(G)$ is the enveloping $\mathrm{C}^{*}$-algebra of $\mathcal{R}(G)$.

Proposition 5.4. Let $G$ be a compact group and let $\pi: \mathcal{R}(G) \longrightarrow A$ be a *-representation on a $\mathrm{C}^{*}$-algebra $A$. Extend $\pi$ to a $*$-algebra map $\pi^{+}: C(G) \longrightarrow A$, and let $X$ be the spectrum of $\pi^{+}(C(G))$, so that $\pi^{+}(C(G)) \simeq C(X)$ and that the induced $\mathrm{C}^{*}$ algebra map $C(G) \longrightarrow \pi^{+}(C(G)) \simeq C(X)$ comes from a continuous map $v: X \longrightarrow G$. Then $\mathcal{R}(G)_{\pi} \simeq \mathcal{R}(\overline{\langle v(X)\rangle})$, where $\overline{\langle v(X)\rangle}$ is the closure in $G$ of the subgroup generated by $v(X)$.

Proof. The proof is essentially the same as the one of Proposition 5.1, because a quotient of a compact Hopf algebra is itself compact, and by the Hopf algebra version of the Tannaka-Krein theorem, a commutative compact Hopf algebra is the algebra of representative functions on a unique compact group.

Proposition 5.5. Let $G$ be a compact Lie group. Then there exists an inner faithful *-representation $\pi: \mathcal{R}(G) \longrightarrow \mathbb{C}^{n}$ for some $n \in \mathbb{N}^{*}$.

Proof. If $G$ is connected, it is known (see [5]) that there exists $x, y \in G$ such $G=$ $\overline{\langle x, y\rangle}$. Similarly to Example 5.2, this gives a $*$-representation $\mathcal{R}(G) \longrightarrow \mathbb{C}^{2}$ which is inner faithful by the previous proposition. If $G$ is not connected, let $G_{0}$ be the neutral component of $G$ : the group $G / G_{0}$ is finite and hence $G$ has a family of $2\left[G: G_{0}\right]$ topological generators, which again gives an inner faithful representation on $\mathbb{C}^{n}$, with $n=2\left[G: G_{0}\right]$.

We end the section by showing that the simple example of function algebras shows that it is not possible to deduce inner faithfulness of a representation of a co-semisimple Hopf algebra by only studying its restriction to characters.

Let us assume that $k$ is algebraically closed. Recall that a co-semi-simple Hopf algebra is a Hopf algebra $H$, whose category of comodules is semi-simple. This is equivalent to say that $H$ has a Peter-Weyl decomposition

$$
H=\bigoplus_{\lambda \in \Lambda} H_{\lambda}
$$

where $\Lambda$ is the set of simple $H$-comodules and for $\lambda \in \Lambda, H_{\lambda}$ is the comatrix coalgebra of corresponding coefficients. Let $d_{\lambda}$ be the dimension of the simple $H$-comodule corresponding to $\lambda$, and let $\chi_{\lambda} \in H_{\lambda}$ be the corresponding character.

The group algebra case corresponds to when $d_{\lambda}=1$ for any $\lambda$, and then the characters correspond to the group-like elements. Since for group algebras inner faithfulness can be detected by only studying the restriction of a representation to group-like elements, it would be natural to hope that in the general case, the restriction to characters would lead to the same information. The following simple example shows that this is not true.

EXAMPLE 5.6. Let us consider $S_{4}$, the symmetric group on 4 letters, and let $\mathbb{C}\left(S_{4}\right)=$ $\mathbb{C}\left[S_{4}\right]^{*}$ be the co-semi-simple Hopf algebra of (complex) functions on $S_{4}$. The group $S_{4}$ is generated by the elements $\tau_{1}=(1,2), \tau_{2}=(2,3)$ and $\tau_{3}=(3,4)$, and hence we have an inner faithful representation

$$
\begin{aligned}
\mathbb{C}\left(S_{4}\right) & \longrightarrow \mathbb{C}^{3} \\
f & \longmapsto\left(f\left(\tau_{1}\right), f\left(\tau_{2}\right), f\left(\tau_{3}\right)\right) .
\end{aligned}
$$


The elements $\tau_{1}, \tau_{2}$ and $\tau_{3}$ are all conjugate in $S_{4}$, so this representation is not injective on characters. Thus an inner faithful representation is not necessarily injective on characters.

Conversely, the representation

$$
\begin{aligned}
\mathbb{C}\left(S_{4}\right) & \longrightarrow \mathbb{C}^{2} \\
f & \longmapsto\left(f\left(\tau_{1}\right), f((1,2,3))\right)
\end{aligned}
$$

is injective on characters (as one easily checks on the character table of $S_{4}$ ), but is not inner faithful since the elements $\tau_{1}$ and $(1,2,3)$ do not generate $S_{4}$.

As a conclusion, it seems that there is no link between the inner faithfulness of a representation and its injectivity on characters.

6. Twisting. In this section, we study the behaviour of Hopf images under various deformation procedures such as Drinfeld's twisting, or the dual operation, often called 2-cocycle deformation, which we will call here cotwisting for simplicity.

6.1. Twisting. We begin with the twisting operation, in the sense of Drinfeld [16]. More exactly, the definitions we use are taken or adapted from $[\mathbf{1 7}, \mathbf{2 3}]$.

Let $H$ be a Hopf algebra and let $\Omega$ be an invertible element in $H \otimes H$. Consider the linear maps $\delta_{\Omega}, \Delta_{\Omega}: H \longrightarrow H \otimes H$ defined respectively by

$$
\delta_{\Omega}(x)=\Omega \Delta(x), \quad \Delta_{\Omega}(x)=\Omega \Delta(x) \Omega^{-1} .
$$

We say that $\Omega$ is a twist on $H$ if

$$
\Omega_{12}\left(\Delta \otimes 1_{H}\right)(\Omega)=\Omega_{23}\left(1_{H} \otimes \Delta\right)(\Omega), \quad\left(\varepsilon \otimes 1_{H}\right)(\Omega)=1=\left(1_{H} \otimes \varepsilon\right)(\Omega)
$$

which is equivalent to say that $\left(H, \delta_{\Omega}, \varepsilon\right)$ is a coalgebra. The element $u=m \circ\left(\mathrm{id}_{\mathrm{H}} \otimes\right.$ $S)(\Omega)$ is then invertible in $H$, and $H_{\Omega}=\left(H, m, u, \Delta_{\Omega}, \varepsilon, S_{u}\right)$ is a Hopf algebra, where $S_{u}: H \longrightarrow H$ is defined by $S_{u}(x)=u S(x) u^{-1}$.

We say that $\Omega$ is a pseudo-twist on $H$ if $(\varepsilon \otimes \mathrm{id})(\Omega)=1=(\mathrm{id} \otimes \varepsilon)(\Omega)$, if $\left(H, \Delta_{\Omega}, \varepsilon\right)$ is a coalgebra, and if there exists an invertible element $u \in H$ such that $S_{u}: H \longrightarrow H$, defined by $S_{u}(x)=u S(x) u^{-1}$, is an antipode for the bialgebra $\left(H, m, u, \Delta_{\Omega}, \varepsilon\right)$, so that $H_{\Omega}=\left(H, m, u, \Delta_{\Omega}, \varepsilon, S_{u}\right)$ is a Hopf algebra.

A Hopf algebra having the form $H_{\Omega}$ for some twist (resp. pseudo-twist) $\Omega$ on $H$ is said to be a twist (resp. pseudo-twist) of $H$.

The following lemma gives some basic properties of twisting, probably well known. The direct verification is left to the reader.

LemMa 6.1. Let $\Omega$ be a pseudo-twist on a Hopf algebra $H$.

(1) Let $f: H \longrightarrow$ L be a surjective Hopf algebra map. Then $f^{*}(\Omega)=(f \otimes f)(\Omega)$ is also a pseudo-twist for $L$.

(2) The Hopf ideals in $H$ are exactly the Hopf ideals in $H_{\Omega}$.

THEOREM 6.2. Let $H$ be a Hopf algebra, let $\Omega$ be a pseudo-twist on $H$ and let $\pi: H \longrightarrow$ A be a representation on an algebra $A$, that we also view as a representation $\pi$ : $H_{\Omega} \longrightarrow$ A. We have $\left(H_{\Omega}\right)_{\pi}=\left(H_{\pi}\right)_{p^{*}(\Omega)}$, where $p: H \longrightarrow H_{\pi}$ is the canonical projection, and $\pi$ is inner faithful as a representation of $H$ if and only if it is inner faithful as a representation of $H_{\Omega}$. 
Proof. Since by the previous lemma the Hopf ideals of $H$ and of $H_{\Omega}$ are the same, this is also true for the Hopf ideals contained in $\operatorname{Ker}(\pi)$, and the largest one is the same. So the defining Hopf ideal of $\left(H_{\Omega}\right)_{\pi}$ is the Hopf ideal $I_{\pi}$ constructed in Section 2, so that $\left(H_{\Omega}\right)_{\pi}=H_{\Omega} / I_{\pi}$, and since $\left(H / I_{\pi}\right)_{p^{*}}(\Omega)=H_{\Omega} / I_{\pi}$, we have the claimed result. The last assertion is also immediate after the previous discussion.

It follows that the twist of an inner linear Hopf algebra is still inner linear. The reader will find several examples of twisted Hopf algebras in [17, 23], for example, for which the above theorem furnishes inner faithful representations of the new Hopf algebra from the old one. Some of the Hopf algebras considered in Section 9 are also obtained by twisting.

6.2. Cotwisting. We now deal with the dual operation to twisting, which we call cotwisting. The situation is more complicated here, because we deform the product rather than the coproduct, and so a representation of the given Hopf algebra is not a representation of the deformed one.

Let us recall the basic vocabulary, which is dual to the one of the previous subsection. We only consider the cotwist case.

Let $H=H$ be a Hopf algebra. We use Sweedler's notation $\Delta(x)=x_{1} \otimes x_{2}$. Recall (see e.g. [15]) that a cotwist $(=2$-cocycle) is a convolution invertible linear map $\sigma$ : $H \otimes H \longrightarrow k$ satisfying

$$
\sigma\left(x_{1}, y_{1}\right) \sigma\left(x_{2} y_{2}, z\right)=\sigma\left(y_{1}, z_{1}\right) \sigma\left(x, y_{2} z_{2}\right)
$$

and $\sigma(x, 1)=\sigma(1, x)=\varepsilon(x)$, for $x, y, z \in H$.

Following [15, 24], we associate various algebras to a cotwist. First, consider the algebra ${ }_{\sigma} H$. As a vector space we have ${ }_{\sigma} H=H$ and the product of ${ }_{\sigma} H$ is defined to be

$$
\{x\}\{y\}=\sigma\left(x_{1}, y_{1}\right)\left\{x_{2} y_{2}\right\}, \quad x, y \in H,
$$

where an element $x \in H$ is denoted $\{x\}$, when viewed as an element of ${ }_{\sigma} H$.

We also have the algebra $H_{\sigma^{-1}}$, where $\sigma^{-1}$ denotes the convolution inverse of $\sigma$. As a vector space we have $H_{\sigma^{-1}}=H$ and the product of $H_{\sigma^{-1}}$ is defined to be

$$
\langle x\rangle\langle y\rangle=\sigma^{-1}\left(x_{2}, y_{2}\right)\left\langle x_{1} y_{1}\right\rangle, \quad x, y \in H,
$$

where an element $x \in H$ is denoted $\langle x\rangle$, when viewed as an element of $H_{\sigma^{-1}}$. The cocycle condition ensures that ${ }_{\sigma} H$ and $H_{\sigma^{-1}}$ are associative algebras with 1 as a unit.

Finally, we have the Hopf algebra $H^{\sigma}={ }_{\sigma} H_{\sigma^{-1}}$. As a coalgebra $H^{\sigma}=H$. The product of $H^{\sigma}$ is defined to be

$$
[x][y]=\sigma\left(x_{1}, y_{1}\right) \sigma^{-1}\left(x_{3}, y_{3}\right)\left[x_{2} y_{2}\right], \quad x, y \in H,
$$

where an element $x \in H$ is denoted [x], when viewed as an element of $H^{\sigma}$, and we have the following formula for the antipode of $H^{\sigma}$ :

$$
S^{\sigma}([x])=\sigma\left(x_{1}, S\left(x_{2}\right)\right) \sigma^{-1}\left(S\left(x_{4}\right), x_{5}\right)\left[S\left(x_{3}\right)\right] .
$$

A Hopf algebra having the form $H^{\sigma}$ for some cotwist $\sigma$ on $H$ is said to be a cotwist of $H$. 
Very often cotwists are induced by simpler quotient Hopf algebras. More precisely let $\pi: H \rightarrow K$ be a Hopf algebra surjection and let $\sigma: K \otimes K \rightarrow \mathbb{C}$ be a cotwist on $K$. Then $\sigma_{\pi}=\sigma \circ(\pi \otimes \pi): H \otimes H \rightarrow \mathbb{C}$ is a cotwist.

We prove the following result.

THEOREM 6.3. Let $H$ be a Hopf algebra and let $\sigma: H \otimes H \rightarrow k$ be a cotwist induced by a finite-dimensional quotient Hopf algebra of $H$. Assume that $S^{2}$ is an inner automorphism of $H$. If $H$ is inner linear, then $H^{\sigma}$ is also inner linear.

The proof will be a consequence of the following technical result.

THEOREM 6.4. Let $\theta: H \longrightarrow A \otimes L$ be an algebra map, where $H, L$ are Hopf algebras and $A$ is an algebra, and let $\varphi: L \longrightarrow B$ be an inner faithful representation of $L$ on an algebra $B$. Assume that there exists $\psi \in A^{*}$ such that $\left(\psi \otimes \mathrm{id}_{L}\right) \circ \theta: H \longrightarrow L$ is an injective coalgebra map. Assume moreover that one of the following conditions holds.

(1) $S_{L} \circ\left(\left(\psi \otimes \mathrm{id}_{L}\right) \circ \theta\right)=\left(\left(\psi \otimes \mathrm{id}_{L}\right) \circ \theta\right) \circ S_{H}$.

(2) $S_{L}(\operatorname{Ker}(\varphi)) \subset \operatorname{Ker}(\varphi)$.

Then the representation $\left(\mathrm{id}_{A} \otimes \varphi\right) \circ \theta: H \longrightarrow A \otimes B$ is inner faithful.

Proof. Let $I \subset \operatorname{Ker}\left(\left(\mathrm{id}_{A} \otimes \varphi\right) \circ \theta\right)$ be a Hopf ideal. Let $J=\left(\psi \otimes \mathrm{id}_{L}\right)(\theta(I)) \subset L$ : this is a co-ideal since $\left(\psi \otimes \mathrm{id}_{L}\right) \circ \theta$ is a coalgebra map. We have

$$
\varphi(J)=(\psi \otimes \varphi)(\theta(I))=\left(\psi \otimes \mathrm{id}_{L}\right)\left(\left(\mathrm{id}_{A} \otimes \varphi\right)(\theta(I))=0,\right.
$$

and hence $J \subset \operatorname{Ker}(\varphi)$. Let $J^{\prime}$ be the ideal of $L$ generated by $J$ : we have $J^{\prime} \subset \operatorname{Ker}(\varphi)$ and $J^{\prime}$ is a bi-ideal in $L$.

Assume that condition (1) holds. Then $J$ is $S_{L}$-stable and hence so is $J^{\prime}$, which is a Hopf ideal. Since $\varphi$ is inner faithful, we have $J^{\prime}=J=(0)$, and we conclude that $I=(0)$ by the injectivity of $\left(\psi \otimes \mathrm{id}_{L}\right) \circ \theta$.

Assume now that condition (2) holds. Then $J^{\prime}=(0)=J$ by Proposition 2.9, and $I=(0)$, again by the injectivity of $\left(\psi \otimes \mathrm{id}_{L}\right) \circ \theta$.

Proof of Theorem 6.3 By the assumption, there is a Hopf algebra surjection $\pi$ : $H \rightarrow K$ onto a finite-dimensional Hopf algebra $K$ and a cotwist $\tau: K \otimes K \rightarrow k$ such that $\sigma=\tau_{\pi}$. As noted in [8], we have an injective algebra map

$$
\begin{aligned}
\theta: H^{\tau_{\pi}} & \longrightarrow{ }_{\tau} K \otimes K_{\tau^{-1}} \otimes H, \\
{[x] } & \longmapsto\left\{\pi\left(x_{1}\right)\right\} \otimes\left\langle\pi\left(x_{3}\right)\right\rangle \otimes x_{2} .
\end{aligned}
$$

Consider the linear map $\psi=\varepsilon \otimes \varepsilon:{ }_{\tau} K \otimes K_{\tau^{-1}} \longrightarrow k$. We have $(\psi \otimes \mathrm{id}) \circ \theta=\mathrm{id}$, and hence is a coalgebra map. Let $\varphi_{0}: H \longrightarrow B$ be an inner faithful finite-dimensional representation, and let $\varphi: H \longrightarrow B \times B^{\mathrm{op}}, x \longmapsto\left(\varphi_{0}(x), \varphi_{0}(S(x))\right)$. It is clear that $\varphi$ is still inner faithful, and the second condition in the previous theorem is satisfied since $S^{2}$ is inner. Hence the previous theorem ensures that the representation (id $\otimes \varphi$ ) $\circ \theta$ : $H \longrightarrow{ }_{\tau} K \otimes K_{\tau^{-1}} \otimes\left(B \times B^{\text {op }}\right)$ is inner faithful, and we are done since ${ }_{\tau} K \otimes K_{\tau^{-1}}$ is finite-dimensional.

To illustrate the previous theorem, let us examine the case of multi-parametric deformations of $\mathrm{GL}_{n}$ of [4]. We assume that the base field is $\mathbb{C}$ until the end of the section. Let $\mathbf{p}=\left(p_{i j}\right) \in M_{n}(\mathbb{C})$ be a multiplicatively anti-symmetric matrix: $p_{i i}=1$ and $p_{i j} p_{j i}=1$ for all $i, j$. The algebra $\mathcal{O}_{\mathbf{p}}\left(\mathrm{GL}_{n}(\mathbb{C})\right)$ is the algebra presented by generators $x_{i j}$, 
$y_{i j}, 1 \leq i, j \leq n$ submitted to the following relations $(1 \leq i, j, k, l \leq n)$ :

$$
\begin{gathered}
x_{k l} x_{i j}=p_{k i} p_{j l} x_{i j} x_{k l}, \quad y_{k l} y_{i j}=p_{k i} p_{j l} y_{i j} y_{k l}, \quad y_{k l} x_{i j}=p_{i k} p_{l j} x_{i j} y_{k l}, \\
\sum_{k=1}^{n} x_{i k} y_{j k}=\delta_{i j}=\sum_{k=1}^{n} x_{k i} y_{k j} .
\end{gathered}
$$

The presentation we have given avoids the use of the quantum determinant. The algebra $\mathcal{O}_{\mathbf{p}}\left(\mathrm{GL}_{n}(\mathbb{C})\right)$ has a standard Hopf algebra structure, described as follows:

$$
\Delta\left(x_{i j}\right)=\sum_{k} x_{i k} \otimes x_{k j}, \Delta\left(y_{i j}\right)=\sum_{k} y_{i k} \otimes y_{k j}, \varepsilon\left(x_{i j}\right)=\delta_{i j}=\varepsilon\left(y_{i j}\right), S\left(x_{i j}\right)=y_{j i}, S\left(y_{i j}\right)=x_{j i} .
$$

When $p_{i j}=1$ for all $i, j$, one gets the usual Hopf algebra $\mathcal{O}\left(\mathrm{GL}_{n}(\mathbb{C})\right)$. It is known that $\mathcal{O}_{\mathbf{p}}\left(\mathrm{GL}_{n}(\mathbb{C})\right)$ is a cotwist of $\mathcal{O}\left(\mathrm{GL}_{n}(\mathbb{C})\right)$, by a cotwist induced by the quotient Hopf algebra $\mathcal{O}\left(\left(\mathbb{C}^{\times}\right)^{n}\right) \simeq \mathbb{C}\left[\mathbb{Z}^{n}\right]$ (see e.g. [4]).

COROLlaRY 6.5. Let $\mathbf{p}=\left(p_{i j}\right) \in M_{n}(\mathbb{C})$ be a multiplicatively anti-symmetric matrix whose entries are roots of unity. Then the Hopf algebra $\mathcal{O}_{\mathbf{p}}\left(\mathrm{GL}_{n}(\mathbb{C})\right)$ is inner linear.

Proof. Let $M$ be the (finite and cyclic) subgroup of $\mathbb{C}^{\times}$generated by the elements $p_{i j}$. The cotwist defining $\mathcal{O}_{\mathbf{p}}\left(\mathrm{GL}_{n}(\mathbb{C})\right)$ is induced by the quotient Hopf algebra $\mathcal{O}\left(M^{n}\right)$, and thus Theorem 6.3 gives the result.

When the multiplicatively anti-symmetric matrix $\mathbf{p}$ satisfies some appropriate additional conditions, we have similar deformations for various algebraic subgroups of $\mathrm{GL}_{n}$ and Theorem 6.6 holds true with similar proof. For example if $p_{i j}=-1$, $\forall i \neq j$, one gets a Hopf algebra deformation of $\mathcal{O}(\mathrm{O}(n, \mathbb{C}))$, found in $[9$, Section 4$]$ and corresponding to the quantum symmetry group of the hypercube, which therefore is inner linear.

There are, however, interesting situations for which Theorem 6.3 is not sufficient to ensure inner linearity, and where we need the full strength of Theorem 6.4, for example, for the Hopf algebra $\mathcal{O}_{-1}\left(S L_{2}(\mathbb{C})\right.$ ) (which is well known not to be a cotwist of $\left.\mathcal{O}\left(S L_{2}(\mathbb{C})\right)\right)$.

COROLlaRY 6.6. The Hopf algebra $\mathcal{O}_{-1}\left(S L_{n}(\mathbb{C})\right)$ is inner linear for any $n \geq 1$.

Proof. The case $n=1$ is trivial while if $n \geq 3$, similarly to the previous cases, $\mathcal{O}_{-1}\left(S L_{n}(\mathbb{C})\right)$ is a cotwist of $\mathcal{O}\left(S L_{n}(\mathbb{C})\right)$ induced by a finite-dimensional quotient. So we assume that $n=2$. As in [28], we have an algebra embedding

$$
\begin{gathered}
\theta: \mathcal{O}_{-1}\left(S L_{2}(\mathbb{C})\right) \hookrightarrow M_{2}(\mathbb{C}) \otimes \mathcal{O}\left(S L_{2}(\mathbb{C})\right), \\
a \mapsto \sigma_{1} \otimes a, b \mapsto \sigma_{2} \otimes b, c \mapsto \sigma_{2} \otimes c, d \mapsto \sigma_{1} \otimes d,
\end{gathered}
$$

where

$$
\sigma_{1}=\left(\begin{array}{ll}
0 & 1 \\
1 & 0
\end{array}\right), \quad \sigma_{2}=\left(\begin{array}{cc}
i & 0 \\
0 & -i
\end{array}\right)
$$

and $a, b, c, d$ denote the standard generators of both $\mathcal{O}\left(S L_{2}(\mathbb{C})\right)$ and $\mathcal{O}_{-1}\left(S L_{2}(\mathbb{C})\right)$. Let $\psi: M_{2}(\mathbb{C}) \rightarrow \mathbb{C}$ be the unique linear map such that $\psi(1)=1=\psi\left(\sigma_{1}\right)=\psi\left(\sigma_{2}\right)=$ 
$\psi\left(\sigma_{1} \sigma_{2}\right)$. It is clear that $(\psi \otimes \mathrm{id}) \circ \theta$ is a vector space isomorphism $\mathcal{O}_{-1}\left(S L_{2}(\mathbb{C})\right) \longrightarrow$ $\mathcal{O}\left(S L_{2}(\mathbb{C})\right)$, and it is not difficult to check that it is a coalgebra map. Now choose topological generators $x, y$ of $S L_{2}(\mathbb{C})$ and consider the inner faithful representation (see Example 5.2)

$$
\varphi: \mathcal{O}\left(S L_{2}(\mathbb{C})\right) \rightarrow \mathbb{C}^{4}, f \mapsto\left(f(x), f(y), f\left(x^{-1}\right), f\left(y^{-1}\right)\right) .
$$

The second condition in Theorem 6.4 is satisfied, and hence we get an inner faithful representation $\mathcal{O}_{-1}\left(S L_{2}(\mathbb{C})\right) \rightarrow M_{2}(\mathbb{C}) \otimes \mathbb{C}^{4}$.

7. Tensor and free product of representations. The Hopf image does not behave well with respect to the tensor or free product: the Hopf image of a tensor or free product is not necessarily the tensor or free product of the Hopf images. Here is what can be said in general.

Proposition 7.1. Let $H$ and $L$ be Hopf algebras and let $\pi: H \longrightarrow A$ and $\varphi: L \longrightarrow$ $B$ be algebra maps. Then there are surjective Hopf algebra maps

$$
H_{\pi} \otimes L_{\varphi} \longrightarrow(H \otimes L)_{\pi \otimes \varphi} \quad \text { and } \quad H_{\pi} * L_{\varphi} \longrightarrow(H * L)_{\pi * \varphi} .
$$

Proof. We consider the Hopf algebra factorizations
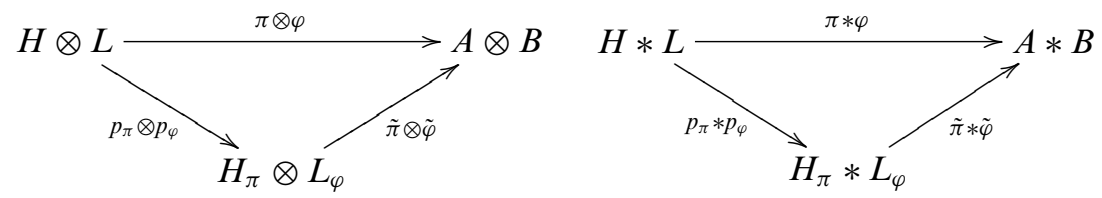

and the universal property of the Hopf image yields the announced Hopf algebra maps, which are clearly surjective.

The morphisms in the proposition are not injective in general, as the following example shows.

EXAMPLE 7.2. Let $\mathbb{Z}_{n}$ be the cyclic group of order $n$ and let $x \in \mathbb{Z}_{n}$ be a generator. Let $\pi: \mathbb{C}\left[\mathbb{Z}_{n}\right] \longrightarrow \mathbb{C}$ be the unique algebra map with $\pi(x)=\omega$, where $\omega$ is a primitive root of unity of order $n$. The representation $\pi$ is inner faithful because its restriction to $\mathbb{Z}_{n}$ is faithful, hence $\mathbb{C}\left[\mathbb{Z}_{n}\right]_{\pi}=\mathbb{C}\left[\mathbb{Z}_{n}\right]$. However, the representations $\pi \otimes \pi$ and $\pi * \pi$ are not inner faithful because the groups $\mathbb{Z}_{n} \times \mathbb{Z}_{n}$ and $\mathbb{Z}_{n} * \mathbb{Z}_{n}$ do not inject in $\mathbb{C}^{*}$.

For the tensor product, a faithfulness assumption on one of the algebra morphisms enables one to describe the Hopf image easily. We begin with a lemma. We use the notation of Section 2.

Lemma 7.3. Let $H$ and $L$ be Hopf algebras and let $\pi: H \longrightarrow A$ and $\varphi: L \longrightarrow B$ be algebra maps. Then

$$
I_{\pi \otimes \varphi}=\bigcap_{g \in F} \operatorname{Ker}\left(\pi^{g} \otimes \varphi^{g}\right)
$$

Proof. We first show that for any $g \in F$, there exists a linear isomorphism $T_{g}$ : $A^{g} \otimes B^{g} \longrightarrow(A \otimes B)^{g}$ such that $(\pi \otimes \varphi)^{g}=T_{g} \circ\left(\pi^{g} \otimes \varphi^{g}\right)$. We prove this by induction 
on $l(g)$. This is clear if $l(g) \leq 1$, so we assume that $g=h h^{\prime}$ with $l(g)>l(h) \geq 1$ and $l(g)>l\left(h^{\prime}\right) \geq 1$. Let $x \in H$ and $y \in L$. We have, using the induction assumption,

$$
\begin{aligned}
(\pi \otimes \varphi)^{h h^{\prime}}(x \otimes y) & =(\pi \otimes \varphi)^{h}\left(x_{1} \otimes y_{1}\right) \otimes(\pi \otimes \varphi)^{h^{\prime}}\left(x_{2} \otimes y_{2}\right) \\
& =\left(T_{h} \otimes T_{h^{\prime}}\right)\left(\pi^{h}\left(x_{1}\right) \otimes \varphi^{h}\left(y_{1}\right) \otimes \pi^{h^{\prime}}\left(x_{2}\right) \otimes \varphi^{h^{\prime}}\left(y_{2}\right)\right) \\
& =T_{h h^{\prime}} \circ\left(\pi^{h h^{\prime}} \otimes \varphi^{h h^{\prime}}\right)(x \otimes y),
\end{aligned}
$$

and hence we have the desired result. Thus for $g \in F$, we have $\operatorname{Ker}\left((\pi \otimes \varphi)^{g}\right)=$ $\operatorname{Ker}\left(\pi^{g} \otimes \varphi^{g}\right)$, and we have our result.

Proposition 7.4. Let $H$ and $L$ be Hopf algebras and let $\pi: H \longrightarrow A$ and $\varphi: L \longrightarrow$ $B$ be algebra maps. Assume that $\pi$ is faithful and that the antipode of $H$ is injective. Then $(H \otimes L)_{\pi \otimes \varphi} \cong H \otimes L_{\varphi}$. In particular, if the antipode of $H$ is injective, if $\pi$ is faithful and if $\varphi$ is inner faithful, then $\pi \otimes \varphi$ is inner faithful.

Proof. It is sufficient to show that $I_{\pi \otimes \varphi}=H \otimes I_{\varphi}$, since $(H \otimes L) /\left(H \otimes I_{\varphi}\right) \simeq H \otimes$ $\left(L / I_{\varphi}\right)$. For any $g \in F$, the representation $\pi^{g}$ is faithful since $\Delta$ and $S$ are injective, so $\operatorname{Ker}\left(\pi^{g} \otimes \varphi^{g}\right)=H \otimes \operatorname{Ker}\left(\varphi^{g}\right)$. By the previous lemma we have

$$
\begin{aligned}
I_{\pi \otimes \varphi} & =\bigcap_{g \in F} \operatorname{Ker}\left(\pi^{g} \otimes \varphi^{g}\right) \\
& =\bigcap_{g \in F} H \otimes \operatorname{Ker}\left(\varphi^{g}\right)=H \otimes\left(\bigcap_{g \in F} \operatorname{Ker}\left(\varphi^{g}\right)\right) \\
& =H \otimes I_{\varphi},
\end{aligned}
$$

and we have our result. The last assertion is immediate.

REMARK 7.5. Of course the assumption of the injectivity of the antipode is satisfied in the most interesting cases. See [25] for an example of a Hopf algebra having a noninjective antipode.

The result in the previous proposition naturally leads to the following question.

QUESTION 7.6. Let $\pi: H \longrightarrow A$ be a representation of a Hopf algebra $H$ on an algebra $A$. Does there exist a condition on $\pi$, weaker than faithfulness, that ensures that for any inner faithful representation $\varphi: L \longrightarrow B$ of a Hopf algebra $L$ on an algebra $B$, then the representation $\pi \otimes \varphi$ is inner faithful?

If a representation $\pi$ satisfies to the hypothetic condition of Question 7.6, then in particular $\pi \otimes \pi$ will be inner faithful. It seems to be simpler to study first representations with this weaker property, and this leads to the following definition.

Definition 7.7. Let $\pi: H \longrightarrow A$ be a representation of a Hopf algebra $H$ on an algebra $A$. We say that $\pi$ is projectively inner faithful if $\pi \otimes \pi$ is inner faithful.

Once again this terminology is motivated by the discrete group case: a representation $\pi: k[\Gamma] \longrightarrow A$ is projectively inner faithful if and only if the associated group morphism $\Gamma \longrightarrow A^{\times} / k^{*}$ is faithful (this follows from the following fact: an element $a \otimes b \in A \otimes B$ in a tensor product of algebras satisfies $a \otimes b=1 \otimes 1$ if and only if $a, b \in k 1$ and $a b=1)$. When $A=\operatorname{End}(V)$, this means that $\pi$ induces an embedding of $\Gamma$ into the projective linear group $\operatorname{PGL}(V)$. 
The example of function algebras shows that it is, in general, difficult to decide when an inner faithful representation is projectively inner faithful. This specific example leads to the following definition. This is a purely discrete group theoretic definition, but of course we have in mind discrete groups embedded as dense subgroups of algebraic groups.

Definition 7.8. Let $\Gamma$ be a discrete group and let $\left\{s_{1}, \ldots, s_{n}\right\}$ be a family of generators of $\Gamma$. We say that $\left\{s_{1}, \ldots, s_{n}\right\}$ is a family of projective generators of $\Gamma$ if the group $\Gamma \times \Gamma$ is generated by the elements $\left(s_{i}, s_{j}\right), 1 \leq i, j \leq n$.

If $\Gamma=\left\langle s_{1}, \ldots, s_{n}\right\rangle$ is a dense subgroup of an algebraic group $G$, then $\left\{s_{1}, \ldots, s_{n}\right\}$ is a family of projective generators of $\Gamma$ if and only if the corresponding representation $\mathcal{O}(G) \longrightarrow \mathbb{C}^{n}$ of Example 5.2 is projectively inner faithful.

Of course $\left\{s_{1}, \ldots, s_{n}\right\}$ is a family of projective generators if $1 \in\left\{s_{1}, \ldots s_{n}\right\}$. It would be interesting to have more examples and to characterize the family of projective generators of a group.

We end the section by noting that there is no analogous result to Proposition 7.4. in the free product case, as shown by the following example.

EXAMPLE 7.9. Let $\pi: \mathbb{C}\left[\mathbb{Z}_{n}\right] \longrightarrow \mathbb{C}$ be the inner faithful representation of Example 7.2. Then $\pi * \mathrm{id}: \mathbb{C}\left[\mathbb{Z}_{n}\right] * \mathbb{C}\left[\mathbb{Z}_{n}\right] \longrightarrow \mathbb{C}\left[\mathbb{Z}_{n}\right]$ is not inner faithful by Proposition 2.12 , since $\mathbb{C}\left[\mathbb{Z}_{n}\right] * \mathbb{C}\left[\mathbb{Z}_{n}\right] \simeq \mathbb{C}\left[\mathbb{Z}_{n} * \mathbb{Z}_{n}\right]$ is a non-commutative algebra while $\mathbb{C}\left[\mathbb{Z}_{n}\right]$ is commutative.

8. Tannaka duality. Tannaka duality studies the interplays between a Hopf algebra and its category of comodules. In this section we formulate some Tannaka type results for Hopf images, providing in this way criterion to prove inner faithfulness of a representation. These results are used in [10], in the study of quantum permutation groups associated to complex Hadamard matrices.

Let $H$ be a Hopf algebra and let $U, V$ be $H$-comodules. The coaction on $U$ is denoted $\alpha_{U}: U \longrightarrow U \otimes H$. The set of $H$-comodule morphisms from $U$ to $V$ is denoted $\operatorname{Hom}_{H}(U, V)$. If $f: H \longrightarrow L$ is a Hopf algebra map, then $f$ induces natural $L$-comodule structures on $U$ and $V$ (the resulting comodules still being denoted $U$ and $V$ if no confusion arises), with

$$
\operatorname{Hom}_{H}(U, V) \subset \operatorname{Hom}_{L}(U, V) .
$$

Now if $\pi: H \longrightarrow A$ is a representation on an algebra $A$ with Hopf image $H_{\pi}$, we have for all $H$-comodules $U, V$,

$$
\operatorname{Hom}_{H}(U, V) \subset \operatorname{Hom}_{H_{\pi}}(U, V) .
$$

The Hopf algebra $H_{\pi}$ is constructed in a very abstract manner in general, but we have a quite concrete description, that just uses $\pi$, for the morphisms between its comodules arising from $H$.

Proposition 8.1. Let $\pi: H \longrightarrow A$ be a representation of a Hopf algebra $H$ on an algebra $A$. Let $U$ and $V$ be finite-dimensional $H$-comodules. We have

$$
\operatorname{Hom}_{H_{\pi}}(U, V)=\operatorname{Hom}\left(U_{\pi}, V_{\pi}\right),
$$


where

$\operatorname{Hom}\left(U_{\pi}, V_{\pi}\right)=\left\{f \in \operatorname{Hom}_{k}(U, V) \mid\left(1_{V} \otimes \pi\right) \circ \alpha_{V} \circ f=\left(1_{V} \otimes \pi\right) \circ\left(f \otimes 1_{H}\right) \circ \alpha_{U}\right\}$.

Proof. Let $f \in \operatorname{Hom}_{H_{\pi}}(U, V)$. We have, with the notations of Theorem 2.1:

$$
\begin{aligned}
& \left(f \otimes 1_{H_{\pi}}\right) \circ\left(1_{U} \otimes p\right) \circ \alpha_{U}=\left(1_{V} \otimes p\right) \circ \alpha_{V} \circ f \\
\Rightarrow & \left(1_{V} \otimes p\right) \circ\left(f \otimes 1_{H}\right) \circ \alpha_{U}=\left(1_{V} \otimes p\right) \circ \alpha_{V} \circ f \\
\Rightarrow & \left(1_{V} \otimes \pi\right) \circ \alpha_{V} \circ f=\left(1_{V} \otimes \pi\right) \circ\left(f \otimes 1_{H}\right) \circ \alpha_{U} .
\end{aligned}
$$

Hence $f \in \operatorname{Hom}\left(U_{\pi}, V_{\pi}\right)$ and $\operatorname{Hom}_{H_{\pi}}(U, V) \subset \operatorname{Hom}\left(U_{\pi}, V_{\pi}\right)$. Assume conversely that $f \in \operatorname{Hom}\left(U_{\pi}, V_{\pi}\right)$, and let $e_{1}, \ldots, e_{m}$ and $e_{1}^{\prime}, \ldots, e_{m}^{\prime}$ be respective bases of $U$ and $V$ with

$$
\alpha_{U}\left(e_{i}\right)=\sum_{k=1}^{n} e_{k} \otimes u_{k i} \quad \text { and } \quad \alpha_{V}\left(e_{j}^{\prime}\right)=\sum_{l=1}^{m} e_{l}^{\prime} \otimes v_{l j} .
$$

Let $\left(\lambda_{i j}\right) \in M_{m, n}(k)$ be such that $f\left(e_{i}\right)=\sum_{j} \lambda_{j i} e_{j}^{\prime}$. Then since $f \in \operatorname{Hom}\left(U_{\pi}, V_{\pi}\right)$, we have for $1 \leq i \leq n, 1 \leq l \leq m$ :

$$
\sum_{j=1}^{m} \pi\left(v_{l j}\right) \lambda_{j i}=\sum_{k=1}^{n} \lambda_{l k} \pi\left(u_{k i}\right)
$$

and hence

$$
P_{l i}:=\sum_{j=1}^{m} v_{l j} \lambda_{j i}-\sum_{k=1}^{n} \lambda_{l k} u_{k i} \in \operatorname{Ker}(\pi) .
$$

Let $I$ be the ideal generated by the elements $P_{l i}$. We have

$$
\Delta\left(P_{l i}\right)=\sum_{j=1}^{m} v_{l j} \otimes P_{j i}+\sum_{k=1}^{n} P_{l k} \otimes u_{k i}
$$

and hence that $I$ is a bi-ideal. Multiplying $P_{i l}$ on the left by $S\left(v_{r l}\right)$ and on the right by $S\left(u_{i s}\right)$ and summing over $i$ and $l$ gives

$$
\sum_{i} \lambda_{r i} S\left(u_{i s}\right)-\sum_{l=1}^{n} S\left(v_{r l}\right) \lambda_{l s}=S\left(-P_{r s}\right) \in I .
$$

Thus $I$ is a Hopf ideal and hence $I \subset I_{\pi}=\operatorname{Ker}(p)$. Thus we have, for $1 \leq i \leq n, 1 \leq$ $l \leq m$ :

$$
\sum_{j=1}^{m} p\left(v_{l j}\right) \lambda_{j i}=\sum_{k=1}^{n} \lambda_{l k} p\left(u_{k i}\right)
$$

This exactly means that $f \in \operatorname{Hom}_{H_{\pi}}(U, V)$, and we are done.

Corollary 8.2. Let $\pi: H \longrightarrow A$ be a representation of a Hopf algebra $H$ on an algebra $A$. 
(1) If $\pi$ is inner faithful, we have

$$
\operatorname{Hom}_{H}(U, V)=\operatorname{Hom}\left(U_{\pi}, V_{\pi}\right)
$$

for any $H$-comodules $U$ and $V$.

(2) Assume that $H$ is co-semi-simple and that the Hopf image $H_{\pi}$ is co-semi-simple. Then $\pi$ is inner faithful if and only if

$$
\operatorname{Hom}_{H}(U, V)=\operatorname{Hom}\left(U_{\pi}, V_{\pi}\right)
$$

for any simple $H$-comodules $U$ and $V$.

Proof. (1) This follows from the previous result.

(2) The $\Rightarrow$ part follows from (1). Conversely, let us assume that

$$
\operatorname{Hom}_{H}(U, V)=\operatorname{Hom}\left(U_{\pi}, V_{\pi}\right)=\operatorname{Hom}_{H_{\pi}}(U, V)
$$

for any simple $H$-comodules $U$ and $V$. Then the canonical projection $p: H \longrightarrow H_{\pi}$ induces an injection from the set of simple $H$-comodules to the set of simple $H_{\pi}$ comodules, and using the respective Peter-Weyl decompositions of $H$ and $H_{\pi}$, we see that $p$ is injective, and hence is an isomorphism.

The converse of part (1) of the corollary is not true in general. To see this, assume that $k$ has characteristic zero and consider $H=\mathcal{O}\left(\mathrm{SL}_{2}(k)\right)$ and $L=\mathcal{O}(B)$, with $B$ being the Borel subgroup of $\mathrm{SL}_{2}(k)$ consisting of triangular matrices. The restriction map $\mathcal{O}\left(\mathrm{SL}_{2}(k)\right) \longrightarrow \mathcal{O}(B)$ is not inner faithful because it is not faithful (by Proposition 2.12), and has $\mathcal{O}(B)$ as Hopf image. One easily sees that for the irreducible representations of $\mathrm{SL}_{2}(k)$ (the symmetric powers of the fundamental representation), one has $\operatorname{Hom}_{\mathrm{SL}_{2}(k)}(U, V)=\operatorname{Hom}_{B}(U, V)$, and hence by the co-semi-simplicity of $\mathcal{O}\left(\mathrm{SL}_{2}(k)\right)$, this is true for any representation of $\mathrm{SL}_{2}(k)$.

It seems to be difficult in general to decide whether the Hopf image is co-semisimple. However this is automatically true when one works in the category of compact Hopf algebras (in the sense of Section 2), and hence we get the following interesting characterization of inner faithfulness for $*$-representations.

THEOREM 8.3. Let $\pi: H \longrightarrow$ A be a $*$-representation of a compact Hopf algebra $H$ on a $*$-algebra $A$. Then $\pi$ is inner faithful if and only if

$$
\operatorname{Hom}_{H}(U, V)=\operatorname{Hom}\left(U_{\pi}, V_{\pi}\right)
$$

for any simple $H$-comodules $U$ and $V$.

Proof. The proof is done by the straightforward adaptation of the arguments of Proposition 8.1 and Corollary 8.2 to the $*$-case.

There is also a useful variant of the above theorem, when $H$ is finitely generated. Let $U$ be a finite-dimensional $H$-comodule. To any $x \in \mathbb{N} * \mathbb{N}=\langle\alpha, \beta\rangle$, we associate an $H$ comodule $U^{x}$ as follows: $U^{1}=\mathbb{C}, U^{\alpha}=U, U^{\beta}=U^{*}, U^{\alpha \beta}=U \otimes U^{*}, U^{\beta \alpha}=U^{*} \otimes U$ and so on. We say that the $H$-comodule $U$ is faithful if any finite finite-dimensional comodule is a sub-quotient of a direct sum of objects of type $U^{x}$. Assuming that $H$ is compact, this is equivalent to saying that any simple $H$-comodule is a sub-object of some $U^{x}$. If $u=\left(u_{i j}\right)$ is the matrix of coefficients of $U$, it is known that $U$ is faithful if and only if the coefficients $u_{i j}$ and $S\left(u_{i j}\right)$ generate $H$ as an algebra. 
TheOREM 8.4. Let $\pi: H \longrightarrow A$ be a $*$-representation of a compact Hopf algebra $H$ on a $*$-algebra $A$, and let $U$ be a faithful $H$-comodule. Then $\pi$ is inner faithful if and only if

$$
\operatorname{Hom}_{H}\left(\mathbb{C}, U^{x}\right)=\operatorname{Hom}\left(\mathbb{C}_{\pi}, U_{\pi}^{x}\right)
$$

for any $x \in \mathbb{N} * \mathbb{N}$. If moreover $U$ is self dual, then $\pi$ is inner faithful if and only if

$$
\operatorname{Hom}_{H}\left(\mathbb{C}, U^{\otimes n}\right)=\operatorname{Hom}\left(\mathbb{C}_{\pi}, U_{\pi}^{\otimes n}\right)
$$

for any $n \in \mathbb{N}$.

Proof. The $\Rightarrow$ part follows from the previous result. Conversely, assume that $\operatorname{Hom}_{H}\left(\mathbb{C}, U^{x}\right)=\operatorname{Hom}\left(\mathbb{C}_{\pi}, U_{\pi}^{x}\right)$ for any $x \in \mathbb{N} * \mathbb{N}$. By duality theory in monoidal categories, we get

$$
\operatorname{Hom}_{H}\left(U^{x}, U^{y}\right)=\operatorname{Hom}\left(U_{\pi}^{x}, U_{\pi}^{y}\right)\left(=\operatorname{Hom}_{H_{\pi}}\left(U^{x}, U^{y}\right)\right)
$$

for any $x, y \in \mathbb{N} * \mathbb{N}$. Hence by Lemma 5.3 in [7] we see that the canonical morphism $p: H \longrightarrow H_{\pi}$ is an isomorphism, which shows that $\pi$ is inner faithful.

If $U$ is self-dual, all the comodules $U^{x}$ are isomorphic with $U^{\otimes n}$ for some $n$, and hence the result follows.

9. Hopf algebras with small corepresentation level. This section gives a concrete application of the inner faithfulness criterion of the previous section to compact Hopf algebras having all their simple comodules of dimension smaller than 2. The Hopf algebras we consider arise in the study of $4 \times 4$ Hadmard matrices $([\mathbf{1 0}, \mathbf{1 2}])$. Let us begin with some vocabulary.

Definition 9.1. Let $H$ be a Hopf algebra and let $n \in \mathbb{N}^{*}$. We say that $H$ has corepresentation level $n$ if there exists a simple $H$-comodule $V$ with $\operatorname{dim}(V)=n$, and if any simple $H$-comodule has dimension smaller or equal than $n$. In this case we put $c l(H)=n$. If the set of dimensions of simple $H$-comodules is not bounded, we put $c l(H)=\infty$.

Pointed Hopf algebras are exactly the Hopf algebras with $\operatorname{cl}(H)=1$. The finite groups $\Gamma$ having all their irreducible representations of dimension $\leq 2$ (in characteristic zero) are described in [1], corresponding to the function Hopf algebras $k^{\Gamma}$ such that $c l\left(k^{\Gamma}\right) \leq 2$.

We begin with a general result (Theorem 9.2) that ensures that a representation of a compact Hopf algebra with corepresentation level 2 is inner faithful. Then this result will be used to construct inner faithful representations of some concrete Hopf algebras in Theorems 9.3 and 9.4 .

THEOREM 9.2. Let $H$ be a compact Hopf algebra with $c l(H)=2$. Let $\Gamma$ be the group of group-like elements of $H$ and let $\Lambda$ be the set of isomorphism classes of simple two-dimensional $H$-comodules. For each $\lambda \in \Lambda$, fix a matrix $u^{\lambda}=\left(u_{i j}^{\lambda}\right) \in M_{2}(H)$ of corresponding coefficients. Let $\pi: H \longrightarrow A$ be $a *$-representation. Assume that the following conditions are fulfilled.

(1) $\pi_{\mid \Gamma}$ is injective.

(2) $\forall \lambda \in \Lambda, \pi\left(u_{11}^{\lambda}\right)=0=\pi\left(u_{22}^{\lambda}\right)$. 
(3) $\forall \lambda \in \Lambda, \forall g \in \Gamma, \pi\left(u_{12}^{\lambda}\right)$ and $\pi(g)$ are linearly independent.

(4) $\forall \lambda, \mu \in \Lambda, \pi\left(u_{12}^{\lambda}\right)$ and $\pi\left(u_{21}^{\mu}\right)$ are linearly independent.

(5) $\forall \lambda, \mu \in \Lambda$ with $\lambda \neq \mu, \pi\left(u_{12}^{\lambda}\right)$ and $\pi\left(u_{12}^{\mu}\right)$ are linearly independent.

Then $\pi$ is inner faithful.

Proof. We have to show, by Theorem 8.3, that $\operatorname{Hom}_{H}(U, V)=\operatorname{Hom}\left(U_{\pi}, V_{\pi}\right)$ for any simple $H$-comodules $U$ and $V$. If $U$ and $V$ have dimension 1 , this is ensured by condition (1). Assume that $U$ has dimension 2, corresponding to $\lambda \in \Lambda$ and that $V$ has dimension 1, corresponding to $g \in \Gamma$. Then $\operatorname{Hom}\left(U_{\pi}, V_{\pi}\right)$ is identified with the spaces of matrices $t=\left(t_{1}, t_{2}\right) \in M_{1,2}(\mathbb{C})$ such that

$$
\left(t_{1}, t_{2}\right)\left(\begin{array}{ll}
\pi\left(u_{11}^{\lambda}\right) & \pi\left(u_{12}^{\lambda}\right) \\
\pi\left(u_{21}^{\lambda}\right) & \pi\left(u_{22}^{\lambda}\right)
\end{array}\right)=\left(t_{1} \pi(g), t_{2} \pi(g)\right) .
$$

Using assumptions (2) and (3), we see that $\operatorname{Hom}\left(U_{\pi}, V_{\pi}\right)=\{0\}=\operatorname{Hom}_{H}(U, V)$. Using a similar argument, we see that the same conclusion holds if $\operatorname{dim}(U)=1$ and $\operatorname{dim}(V)=$ 2. Assume now that $\operatorname{dim}(U)=2=\operatorname{dim}(V)$, corresponding, respectively, to $\lambda, \mu \in \Lambda$. Then $\operatorname{Hom}\left(U_{\pi}, V_{\pi}\right)$ is identified with the space of matrices $t=\left(t_{i j}\right) \in M_{2}(\mathbb{C})$ such that

$$
\left(\begin{array}{ll}
\pi\left(u_{11}^{\mu}\right) & \pi\left(u_{12}^{\mu}\right) \\
\pi\left(u_{21}^{\mu}\right) & \pi\left(u_{22}^{\mu}\right)
\end{array}\right)\left(\begin{array}{ll}
t_{11} & t_{12} \\
t_{21} & t_{22}
\end{array}\right)=\left(\begin{array}{ll}
t_{11} & t_{12} \\
t_{21} & t_{22}
\end{array}\right)\left(\begin{array}{ll}
\pi\left(u_{11}^{\lambda}\right) & \pi\left(u_{12}^{\lambda}\right) \\
\pi\left(u_{21}^{\lambda}\right) & \pi\left(u_{22}^{\lambda}\right)
\end{array}\right) .
$$

Using assumptions (2) and (4), we see that $t_{21}=t_{12}=0$ and if $\lambda=\mu$ we have $t_{11}=t_{22}$, and hence $\operatorname{Hom}\left(U_{\pi}, V_{\pi}\right)=\operatorname{Hom}_{H}(U, V)=\mathbb{C}$. If $\lambda \neq \mu$, we have $t_{11}=t_{22}=0$ by (5) and hence $\operatorname{Hom}\left(U_{\pi}, V_{\pi}\right)=(0)=\operatorname{Hom}_{H}(U, V)$ : this concludes the proof.

We now use Theorem 9.2 to provide inner faithful representations of a class of Hopf algebras considered in [8], Section 7. We refer the reader to [8] for the precise origins of these Hopf algebras, which we present now. First we have the Hopf algebra $A_{h}(2)$ : this is the universal $*$-algebra presented by generators $\left(v_{i j}\right)_{1 \leq i, j \leq 2}$ and relations:

(1) The matrix $v=\left(v_{i j}\right)$ is orthogonal (with $v_{i j}^{*}=v_{i j}$ ).

(2) $v_{i j} v_{i k}=v_{i k} v_{k j}=0=v_{j i} v_{k i}=v_{k i} v_{j i}$ if $j \neq k$.

The standard formulae

$$
\Delta\left(v_{i j}\right)=\sum_{k} v_{i k} \otimes v_{k j}, \quad \varepsilon\left(v_{i j}\right)=\delta_{i j}, S\left(v_{i j}\right)=v_{j i}
$$

endow $A_{h}(2)$ with a compact Hopf algebra structure. We have $\operatorname{cl}\left(A_{h}(2)\right)=2$, and the fusion rules of the corepresentations of $A_{h}(2)$ are those of the orthogonal group $\mathrm{O}(2)$. follows.

The Hopf algebra $A_{h}(2)$ has a series of finite-dimensional quotients defined as

For $m \in \mathbb{N}^{*}$ and $e= \pm 1$, the compact Hopf algebra $A(2 m, e)$ is the quotient of $A_{h}(2)$ by the relations

$$
\left(v_{11} v_{22}\right)^{m}=\left(v_{22} v_{11}\right)^{m},\left(v_{12} v_{21}\right)^{m}=e\left(v_{21} v_{12}\right)^{m} .
$$

Similarly, for $m \in \mathbb{N}$ and $e= \pm 1$, the compact Hopf algebra $A(2 m+1, e)$ is the quotient of $A_{h}(2)$ by the relations

$$
\left(v_{11} v_{22}\right)^{m} v_{11}=\left(v_{22} v_{11}\right)^{m} v_{22},\left(v_{12} v_{21}\right)^{m} v_{12}=e\left(v_{21} v_{12}\right)^{m} v_{21} .
$$

For any $k \in \mathbb{N}^{*}$, we have $\operatorname{dim}(A(k, e))=4 k$ and $\operatorname{cl}(A(k, e))=2$ if $k \geq 2$. 
TheOREM 9.3. Let $q \in \mathbb{C}^{*}$ with $q \neq 1$ and $|q|=1$. Then there exists a unique *-representation $\pi_{q}: A_{h}(2) \longrightarrow M_{2}(\mathbb{C})$ such that

$$
\pi_{q}\left(v_{11}\right)=0=\pi_{q}\left(v_{22}\right), \pi_{q}\left(v_{12}\right)=\left(\begin{array}{cc}
0 & q^{-1} \\
q & 0
\end{array}\right), \pi_{q}\left(v_{21}\right)=\left(\begin{array}{ll}
0 & 1 \\
1 & 0
\end{array}\right) .
$$

(1) If $q$ is not a root of unity, then the $*$-representation $\pi_{q}$ is inner faithful.

(2) If q has order $2 m+1$, then $A_{h}(2)_{\pi_{q}} \simeq A(2 m+1,1)$.

(3) If $q$ has order $4 m$, then $A_{h}(2)_{\pi_{q}} \simeq A(2 m,-1)$.

(4) If q has order $4 m+2$, then $A_{h}(2)_{\pi_{q}} \simeq A(2 m+1,-1)$.

Proof. It is straightforward to check the existence of the $*$-representation $\pi_{q}$. For $m \geq 1$, we have

$$
\begin{gathered}
\pi\left(\left(v_{11} v_{22}\right)^{m}\right)=0=\pi\left(\left(v_{22} v_{11}\right)^{m}\right), \\
\pi\left(\left(v_{12} v_{21}\right)^{m}\right)=\left(\begin{array}{cc}
q^{-m} & 0 \\
0 & q^{m}
\end{array}\right), \pi\left(\left(v_{21} v_{12}\right)^{m}\right)=\left(\begin{array}{cc}
q^{m} & 0 \\
0 & q^{-m}
\end{array}\right) .
\end{gathered}
$$

and for $m \geq 0$ we have

$$
\begin{gathered}
\pi\left(\left(v_{11} v_{22}\right)^{m} v_{11}\right)=0=\pi\left(\left(v_{22} v_{11}\right)^{m}\right) v_{22}, \\
\pi\left(\left(v_{12} v_{21}\right)^{m} v_{12}\right)=\left(\begin{array}{cc}
0 & q^{-m-1} \\
q^{m+1} & 0
\end{array}\right), \pi\left(\left(v_{21} v_{12}\right)^{m} v_{21}\right)=\left(\begin{array}{cc}
0 & q^{m} \\
q^{-m} & 0
\end{array}\right) .
\end{gathered}
$$

(1) We have $\operatorname{cl}\left(A_{h}(2)\right)=2$ and the simple $A_{h}(2)$-comodules are as follows: there is only one non-trivial one-dimensional comodule, corresponding to the group-like $d=$ $v_{11}^{2}-v_{12}^{2}$, and a family of simple two-dimensional comodules $V_{n}, n \geq 1$, corresponding to the simple sub-coalgebras

$$
C(2 m)=\mathbb{C}\left(v_{11} v_{22}\right)^{m} \oplus \mathbb{C}\left(v_{12} v_{21}\right)^{m} \oplus \mathbb{C}\left(v_{21} v_{12}\right)^{m} \oplus \mathbb{C}\left(v_{22} v_{11}\right)^{m}, m \geq 1, \text { and }
$$

$$
C(2 m+1)=\mathbb{C}\left(v_{11} v_{22}\right)^{m} v_{11} \oplus \mathbb{C}\left(v_{12} v_{21}\right)^{m} v_{12} \oplus \mathbb{C}\left(v_{21} v_{12}\right)^{m} v_{21} \oplus \mathbb{C}\left(v_{22} v_{11}\right)^{m} v_{22}, m \geq 0
$$

It is straightforward to check that if $q$ is not a root of unity, the representation $\pi_{q}$ satisfies to the conditions of Theorem 9.2, and hence we have our result.

(2) Assume that $q$ is a root of unity of order $2 m+1$. Then the representation $\pi_{q}$ induces a $*$-representation $\tilde{\pi}_{q}: A(2 m+1,1) \longrightarrow M_{2}(\mathbb{C})$, and we have to show that $\tilde{\pi}_{q}$ is inner faithful. For notational simplicity, the projections of elements of $A_{h}(2)$ in $A(2 m+1,1)$ are denoted by the same symbol. We have $\operatorname{cl}(A(2 m+1,1)) \leq 2$ and the simple $A(2 m+1,1)$-comodules are as follows: there are three non-trivial onedimensional comodules, corresponding to the group-like elements

$$
d=v_{11}^{2}-v_{12}^{2}, g=\left(v_{11} v_{22}\right)^{m} v_{11}+\left(v_{12} v_{21}\right)^{m} v_{12}, h=\left(v_{11} v_{22}\right)^{m} v_{11}-\left(v_{12} v_{21}\right)^{m} v_{12},
$$

a family of $2 m$ simple two-dimensional comodules $V_{1}, \ldots, V_{2 m}$, corresponding to the simple sub-coalgebras $C(k)$ just defined above. It is then a direct verification to check 
that the representation $\tilde{\pi}_{q}$ satisfies to the conditions of Theorem 9.2, and hence is inner faithful.

(3) Assume that $q$ is a root of unity of order $4 m(m \geq 1)$. Then the representation $\pi_{q}$ induces a $*$-representation $\tilde{\pi}_{q}: A(2 m,-1) \longrightarrow M_{2}(\mathbb{C})$, and we have to show that $\tilde{\pi}_{q}$ is inner faithful. We have $c l(A(2 m,-1))=2$ and the simple $A(2 m,-1)$-comodules are as follows: there are three non-trivial one-dimensional comodules, corresponding to the group-like elements

$$
d=v_{11}^{2}-v_{12}^{2}, g=\left(v_{11} v_{22}\right)^{m}+i\left(v_{12} v_{21}\right)^{m}, h=\left(v_{11} v_{22}\right)^{m}-i\left(v_{12} v_{21}\right)^{m},
$$

a family of $(2 m-1)$ simple two-dimensional comodules $V_{1}, \ldots, V_{2 m-1}$, corresponding to the simple sub-coalgebras $C(k)$ defined above. It is a direct verification to check that the representation $\tilde{\pi}_{q}$ satisfies to the conditions of Theorem 9.2, and hence is inner faithful.

(4) Assume finally that $q$ is a root of unity of order $4 m+2(m \geq 1)$. Then the representation $\pi_{q}$ induces a $*$-representation $\tilde{\pi}_{q}: A(2 m+1,-1) \longrightarrow M_{2}(\mathbb{C})$, and we have to show that $\tilde{\pi}_{q}$ is inner faithful. We have $\operatorname{cl}(A(2 m+1,-1)) \leq 2$ and the simple $A(2 m+1,-1)$-comodules are as follows: there are three non-trivial one-dimensional comodules, corresponding to the group-like elements

$$
d=v_{11}^{2}-v_{12}^{2}, g=\left(v_{11} v_{22}\right)^{m} v_{11}+i\left(v_{12} v_{21}\right)^{m} v_{12}, h=\left(v_{11} v_{22}\right)^{m} v_{11}-i\left(v_{12} v_{21}\right)^{m} v_{12},
$$

a family of $2 m$ simple two-dimensional comodules $V_{1}, \ldots, V_{2 m}$, corresponding to the simple sub-coalgebras $C(k)$ defined above. It is again a direct verification to check that the representation $\tilde{\pi}_{q}$ satisfies to the conditions of Theorem 9.2, and hence is inner faithful.

The Hopf algebra $A_{h}(2)$ is in fact a particular case of a construction given in [13, Example 2.5], which we describe now, and the inner faithful representation of the previous theorem has a natural generalization.

Let $\Gamma$ be a group and let $n \in \mathbb{N}^{*}$. Let $A_{n}(\Gamma)$ be the algebra presented by generators $a_{i j}(g), 1 \leq i, j \leq n, g \in \Gamma$, and relations $(1 \leq i, j, k \leq n ; g, h \in \Gamma)$ :

$$
a_{i j}(g) a_{i k}(h)=\delta_{j k} a_{i j}(g h) \quad ; \quad a_{j i}(g) a_{k i}(h)=\delta_{j k} a_{j i}(g h) \quad ; \quad \sum_{l=1}^{n} a_{i l}(1)=1=\sum_{l=1}^{n} a_{l i}(1) .
$$

Then $A_{n}(\Gamma)$ is a compact Hopf algebra, with

$$
a_{i j}(g)^{*}=a_{i j}\left(g^{-1}\right) ; \Delta\left(a_{i j}(g)\right)=\sum_{k=1}^{n} a_{i k}(g) \otimes a_{k j}(g) ; \varepsilon\left(a_{i j}(g)\right)=\delta_{i j} ; S\left(a_{i j}(g)\right)=a_{j i}\left(g^{-1}\right) .
$$

The Hopf algebra $A_{h}(2)$ is $A_{2}\left(\mathbb{Z}_{2}\right)$.

We need some notation to state a generalization of the first part of Theorem 9.3. We consider the group free product $\Gamma * \Gamma$, with the canonical morphisms still denoted $v_{1}, v_{2}: \Gamma \longrightarrow \Gamma * \Gamma$. The canonical involutive group automorphism of $\Gamma * \Gamma$ is denoted by $\tau$, with $\tau \circ v_{1}=v_{2}$ and $\tau \circ v_{2}=v_{1}$.

THEOREM 9.4. Let $\Gamma$ be a group, let $A$ be a $*$-algebra, and let $\pi_{0}: \Gamma * \Gamma \longrightarrow A^{\times}$be a group morphism into the group of unitary elements of $A$, such that $\forall x \in \Gamma * \Gamma \backslash\{1\}$, we have $\pi_{0}(x) \notin \mathbb{C} 1$. Then $\pi_{0}$ induces an inner faithful $*$-representation $\pi: A_{2}(\Gamma) \longrightarrow A$ 
such that

$\forall g \in \Gamma, \pi\left(a_{11}(g)\right)=0=\pi\left(a_{22}(g)\right), \pi\left(a_{12}(g)\right)=\pi_{0}\left(v_{1}(g)\right), \pi\left(a_{21}(g)\right)=\pi_{0}\left(v_{2}(g)\right)$.

In particular, if there exists a group embedding $\Gamma * \Gamma \subset \mathrm{PU}(n)$ for some $n \geq 1$, then the Hopf algebra $A_{2}(\Gamma)$ is inner linear.

Proof. It is a direct verification to check that the above formulae define a *-representation $\pi: A_{2}(\Gamma) \longrightarrow A$. Recall from [13] that we have an algebra isomorphism

$$
\begin{aligned}
A_{2}(\Gamma) & \longrightarrow \mathbb{C}\left[(\Gamma * \Gamma) \times \mathbb{Z}_{2}\right], \\
a_{i j}(g) & \longmapsto v_{i}(g) x_{i j},
\end{aligned}
$$

where if $a$ is the generator of $\mathbb{Z}_{2}, x_{11}=\frac{1+a}{2}=x_{22}$ and $x_{12}=\frac{1-a}{2}=x_{21}$ (of course we simply write $a$ for the element $(1, a)$ of $(\Gamma * \Gamma) \times \mathbb{Z}_{2}$, and so on). We freely use this algebra identification in what follows. Let us now recall the corepresentation theory of $A_{2}(\Gamma)$ (Proposition 2.6 in [13]). We assume of course that the group $\Gamma$ is non-trivial (otherwise the statement in the Theorem is trivial). The only non-trivial group-like element in $A_{2}(\Gamma)$ is $d=a_{11}(1)-a_{12}(1)$, for which $\pi(d)=-1$. To any $x \in \Gamma * \Gamma \backslash\{1\}$ is associated a simple comodule $U_{x}$ with corresponding matrix of coefficients defined by

$$
A_{11}(x)=x x_{11}, A_{12}(x)=x x_{12}, A_{21}(x)=\tau(x) x_{21}, A_{22}(x)=\tau(x) x_{22} .
$$

The comodules $U_{x}$ and $U_{y}$ are isomorphic if and only if $x=y$ or $x=\tau(y)$. Any two-dimensional $A_{2}(\Gamma)$-comodule is isomorphic to some $U_{x}$, and $\operatorname{cl}\left(A_{2}(\Gamma)\right)=2$. For $x \in \Gamma * \Gamma \backslash\{1\}$, we have

$$
\pi\left(A_{11}(x)\right)=0=\pi\left(A_{22}(x)\right), \pi\left(A_{12}(x)\right)=\pi_{0}(x), \pi\left(A_{21}(x)\right)=\pi_{0}(\tau(x)) .
$$

Then it is a straightforward verification to check that the conditions of Theorem 9.2 are fulfilled, using the properties of the group morphism $\pi_{0}$ (using also that $x=1$ if and only if $x=\tau(x)$ ), and we conclude that $\pi$ is inner faithful.

ACKNOWLEDGEMENT. We wish to thank Rupert Yu for helpful discussions on algebraic groups. This work was supported by the ANR project 'Galoisint' BLAN073_183390.

\section{REFERENCES}

1. S. A. Amitsur, Groups with representations of bounded degree II, Illinois J. Math. 5 (1961), 198-205.

2. N. Andruskiewitsch and J. Devoto, Extensions of Hopf algebras, St. Petersburg Math. J. 7 (1996), 17-52.

3. N. Andruskiewitsch and H.-J. Schneider, On the classification of finite-dimensional pointed Hopf algebras, Ann. Math. 171 (2010), 375-417.

4. M. Artin, W. Schelter and J. Tate, Quantum deformations of $G L_{n}$, Commun. Pure Appl. Math. 44 (1991), 879-895.

5. H. Auerbach, Sur les groupes linéaires bornés III, Studia Math. 5 (1935), 43-49.

6. T. Banica, Hopf algebras and subfactors associated to vertex models, J. Funct. Anal. 159 (1998), 243-266. 
7. T. Banica, Representations of compact quantum groups and subfactors, J. Reine Angew. Math. 509 (1999), 167-198.

8. T. Banica and J. Bichon, Quantum groups acting on 4 points, J. Reine Angew. Math. 626 (2009), 75-114.

9. T. Banica, J. Bichon and B. Collins, The hyperoctahedral quantum group, J. Ramanujan Math. Soc. 22 (2007), 345-384.

10. T. Banica, J. Bichon and J.-M. Schlenker, Representations of quantum permutations algebras, J. Funct. Anal. 257 (2009), 2864-2910.

11. T. Banica and S. Moroianu, On the structure of quantum permutation groups, Proc. Amer. Math. Soc. 135 (2007), 21-29.

12. T. Banica and R. Nicoara, Quantum groups and Hadamard matrices, Panamer. Math. J. 17 (2007), 1-24.

13. J. Bichon, Free wreath product by the quantum permutation group, Alg. Rep. Theory 7 (2004), 343-362.

14. W. Chin and I. Musson, The coradical filtration for quantized enveloping algebras. J. Lond. Math. Soc. 53 (1996), 50-62.

15. Y. Doi, Braided bialgebras and quadratic algebras, Commun. Algebra 21 (1993), 17311749.

16. V. G. Drinfeld, Quasi-Hopf algebras, Leningrad Math. J. 1 (1990), 1419-1457.

17. M. Enock and L. Vainerman, Deformation of a Kac algebra by an abelian subgroup, Commun. Math. Phys. 178 (1996), 571-596. 2000).

18. P. de la Harpe, Topics in geometric group theory (University of Chicago Press, Chicago,

19. A. Klimyk and K. Schmüdgen, Quantum groups and their representations, texts and monographs in physics (Springer-Verlag, Berlin, 1997).

20. J. Kustermans and S. Vaes, Locally compact quantum groups in the von Neumann algebraic setting, Math. Scand. 92 (2003), 68-92.

21. S. Montgomery, Hopf algebras and their actions on rings, Amer. Math. Soc. (1993).

22. E. Müller, The coradical filtration of $U_{q}(\mathfrak{g})$ at roots of unity, Commun. Algebra 28 (2000), $1029-1044$.

23. D. Nikshych, $K_{0}$-rings and twisting of finite-dimensional semisimple Hopf algebras, Commun. Algebra 26 (1998), 321-342.

24. P. Schauenburg, Hopf bigalois extensions, Comm. Algebra 24 (1996), 3797-3825.

25. P. Schauenburg, Faithful flatness over Hopf subalgebras: Counterexamples, Lecture Notes Pure Appl. Math. (Dekker) 210 (2000), 331-344.

26. M. Sweedler, Hopf algebras (Benjamin, New York, 1969).

27. S. Vaes, Strictly outer actions of groups and quantum groups, J. Reine Angew. Math. 578 (2005), 147-184.

28. S. Zakrzewski, Matrix pseudogroups associated with anti-commutative plane, Lett. Math. Phys. 21 (1991), 309-321. 